\title{
POTENTIAL THEORETIC ANALYSIS OF A CERTAIN INTEGRAL EQUATION
}

BY

GUNNAR A. BROSAMLER

1. Introduction and summary. Our starting point is the following

Problem (D). Given a measure $\mu$ on the Borel sets of $R^{+}$, the nonnegative real line, such that

$$
\mu(\xi, \xi+1] \leqq 1, \quad \xi \in R^{+},
$$

find a real-valued measurable function $h$ on $R^{+}$which satisfies:

$$
\begin{aligned}
h(\xi) & =\int_{(\xi, \xi+1]} h(\eta) \mu(d \eta), \\
\lim _{\xi \rightarrow \infty} h(\xi) & =1 .
\end{aligned}
$$

Such functions play a role in the theory of slowing down of neutrons [15].

The problem was solved by Slater and Wilf [16] under the following very restrictive assumptions on $\mu$ :

$$
\mu(d \xi)=K(\xi) d \xi, \quad 0<K(\xi) \leqq 1, \quad K(\xi) \uparrow, \quad \lim _{\xi \rightarrow \infty} K(\xi)=1 .
$$

Let $\psi$ on $R^{+}$be defined by successive approximation:

$$
\psi(\xi)=\downarrow \lim _{n \rightarrow \infty} \psi_{n}(\xi)
$$

where $\psi_{0}(\xi) \equiv 1$ and $\psi_{n+1}(\xi)=\int_{(\xi, \xi+1]} \psi_{n}(\eta) \mu(d \eta)$. Slater and Wilf prove

(1.6') THEOREM. Under the hypothesis (1:4)

(a) any function $h$ which is a solution of (1.2) and (1.3) is necessarily equal to $\psi$ (uniqueness);

(b) $\psi$ is a solution of (1.2) and (1.3) iff $\psi \not \equiv 0 ; \psi \not \equiv 0$ iff

$$
\int_{0}^{\infty}\{1-K(\xi)\} d \xi<\infty
$$

For the proof of this theorem in [16] the monotoneity assumption on $K$ is crucial: Under this hypothesis it is easy to see that $\psi$ is monotone, which is not true in the general case. Also, if $\mu(d \xi)=K(\xi) d \xi, \psi$ is absolutely continuous, whereas in general we only know that a real-valued function satisfying (1.2) is right-continuous and has limits from the left.

Received by the editors May 24, 1966. 
To solve Problem (D) in general, in particular to get rid of the unnatural monotoneity assumption imposed on $K$ by Slater and Wilf, a different method is needed. The natural approach is the potential theoretic-probabilistic approach which is suggested by the following observation:

There is a formal analogy on one hand between the solutions of (1.2) and the harmonic functions on some open connected set $D \subseteq R^{n}$, as studied in classical potential theory, on the other hand between the solutions of (1.2) and martingales, as studied in probability theory. In all three cases the functions in question are required to satisfy certain average conditions.

From the potential theoretic point of view Problem (D) appears as an analogue of the classical Dirichlet problem. Indeed it can be put into the framework of a potential theory associated with the following substochastic transition function $P$ on $R^{+}$:

$$
P(\xi, A)=\mu\{(\xi, \xi+1] \cap A\}
$$

for $\xi \in R^{+}, A$ a Borel set of $R^{+}$. Potential theories associated with substochastic transition functions $P$ on some measurable space $(\mathfrak{X}, \mathfrak{l})$ were studied e.g., in [6] as generalizations of classical potential theory. They are concerned with the study of $P$-regular and $P$-superregular functions which appear as the analogues of harmonic and superharmonic functions. We recall the following definitions from [6]:

An extended real-valued measurable function $u$ on $\mathfrak{X}$ is called $P$-superregular if (1) $-\infty<u \leqq \infty$,

(2) for $n \geqq 1, P_{n} u(\xi)=\int u(\eta) P_{n}(\xi, d \eta)$ is finite on $\mathfrak{X}$ and $P u \leqq u$. Here

$$
\begin{aligned}
P_{1}(\xi, A) & =P(\xi, A) ; \\
P_{n+1}(\xi, A) & =\int P_{n}(\eta, A) P(\xi, d \eta) \\
& =\int P(\eta, A) P_{n}(\xi, d \eta) .
\end{aligned}
$$

If $u$ is bounded from below, (2) reduces to

(2') $P u<\infty, P u \leqq u$.

We call $h$ on $\mathfrak{X} P$-regular if $h$ and $-h$ are $P$-superregular; i.e., if

(1) $-\infty<h<\infty$,

(2) $P h=h$.

If $P$ is given by (1.7), then $P u(\xi)=\int_{(\xi, \xi+1]} u(\eta) \mu(d \eta)$, and we see that the $P$-regular functions are just the real-valued solutions of (1.2) and that an extended real-valued function $u$ on $R^{+}$, bounded from below is $P$-superregular iff

$$
\int_{(\xi, \xi+1]} u(\eta) \mu(d \eta) \leqq u(\xi) \text { and } \int_{(\xi, \xi+1]} u(\eta) \mu(d \eta)<\infty .
$$

In view of Problem (D) one is particularly interested in the class of $P$-regular functions (then Theorem (2.3) gives the class of $P$-superregular functions, bounded 
from below) and the behavior at $\infty$ of $P$-superregular functions. In classical potential theory the corresponding problems are closely connected with the concept of Martin boundary. In [6] a boundary theory is given for discrete $\mathfrak{X}$, which is of course not applicable in our case where $\mathfrak{X}=R^{+}$; even in the discrete case there are only few examples where the Martin boundary has actually been identified. It is the purpose of this paper to give a complete potential theoretic analysis of (1.2) and $\left(1.2^{\prime}\right)$, i.e., a complete development of the potential theory associated with $P$ defined by (1.7). Much of this work is of course not necessary to solve the original Problem (D); however our various approaches have independent interest since we present one of the very few fully worked out examples of probabilistic potential theory.

Our main results can be described as follows:

(1) $\psi \not \equiv 0$ iff

$$
\limsup _{\xi \rightarrow \infty} \mu(\xi, \xi+1]>0 \text { and } \int_{(0, \infty)}\{1-\mu(\xi, \xi+1]\} \mu(d \xi)<\infty
$$

(Theorem (4.2)).

(2) Assume $\psi \not \equiv 0$. Then we can introduce a Martin boundary, which will turn out to be trivial, i.e., the point $\infty$ of the one-point-compactification of $R^{+}$(Theorem (5.1)). In analogy to the representation theorem for positive harmonic functions we shall prove that every $P$-regular function which is bounded from below or above, is proportional to $\psi$ (Theorem (4.1)). In analogy to the Fatou theorems for positive harmonic and superharmonic functions we shall prove that $\lim _{\xi \rightarrow \infty} \psi(\xi)=1$ (Theorem (4.14)) and that for any $P$-superregular function $u$, which is bounded from below, there exists a Borel set $A$ of finite $\mu$-measure such that $\lim _{\xi \rightarrow \infty ; \xi \notin A} u(\xi)$ exists and is finite (Corollary (4.16)). This latter theorem will be interpreted as "fine" limit theorem after establishing that the Borel sets which are "thin at $\infty$ " are just the sets of finite $\mu$-measure.

(3) In $\$ 7$ we shall study the dual problem (i.e., the problem of $P$-regular and $P$-superregular measures).

Our results will imply immediately the solution of Problem (D) in the general case. Without any other condition on $\mu$ but $(\mathrm{H})$ we will get

(1.6) THEOREM. (a) Any function $h$ on $R^{+}$satisfying (1.2) and (1.3) is necessarily equal to $\psi$.

(b) $\psi$ satisfies (1.2) and (1.3) iff $\psi \not \equiv 0 ; \psi \not \equiv 0$ iff (A) holds.

Our proofs will be potential theoretic, using heavily the concepts of Green function and reduced function. Where possible we shall give probabilistic interpretations in terms of the Markov process connected with the substochastic transition function $P$.

ACKNOWLEDGEMENT. The results of this paper were submitted in a thesis in partial fulfillment of the requirements for the Ph.D. degree in Mathematics at the 
University of Illinois. The thesis was written under the direction of Professor J. L. Doob, to whom the author is greatly indebted for advice and encouragement.

2. Concepts and results from [6].

1. Reduced function. Let $A \in \mathfrak{A}$; for $\eta_{0} \in \mathfrak{X}, B \in \mathfrak{A}$ let

$$
H_{A}\left(\eta_{0}, B\right)=\sum_{k=0}^{\infty} H_{A}^{(k)}\left(\eta_{0}, B\right)
$$

with

$$
\begin{gathered}
H_{A}^{(0)}\left(\eta_{0}, B\right)=\chi_{A B}\left(\eta_{0}\right), \\
H_{A}^{(k)}\left(\eta_{0}, B\right)=\chi_{\mathfrak{X}-A}\left(\eta_{0}\right) \int_{\mathfrak{X}-A} P\left(\eta_{0}, d \eta_{1}\right) \cdots \\
\cdots \int_{\mathfrak{X}-A} P\left(\eta_{k-2}, d \eta_{k-1}\right) \int_{A B} P\left(\eta_{k-1}, d \eta_{k}\right) \quad(k \geqq 1) .
\end{gathered}
$$

It is clear that $H_{A}^{(k)}$ and $H_{A}$ are substochastic transition functions and that for each $\eta_{0} \in \mathfrak{X}, H_{A}^{(k)}\left(\eta_{0}, \cdot\right)$ and $H_{A}\left(\eta_{0}, \cdot\right)$ concentrate their total measure on $A$.

If $u \geqq 0$ is $P$-superregular it can be proved that

$$
H_{A} u(\xi)=\int u(\eta) H_{A}(\xi, d \eta)=\sum_{k=0}^{\infty} \int u(\eta) H_{A}^{(k)}(\xi, d \eta)
$$

called the reduced function of $u$ with respect to $A$, is the infimum of the nonnegative $P$-superregular functions which majorize $u$ on $A . H_{A} u$ has the following properties:

(1) on $\mathfrak{X}: H_{A} u$ is $P$-superregular, $H_{A} u \leqq u$,

(2) on $A: H_{A} u=u$,

(3) on $\mathfrak{X}-A: H_{A} u=P H_{A} u(\leqq \infty)$.

We point out that the preceding definition is quite in analogy to the one in classical potential theory: If $u>0$ is superharmonic on $D \subseteq R^{n}$, then the infimum $v$ of all superharmonic functions which majorize $u$ on $A \subseteq D$ need not be superharmonic since it need not be lower semicontinuous. The reduced function of $u$ with respect to $A, H_{A} u$, is defined as a certain smoothed version of $v$. It satisfies (1) and, except on a "small" set (2) and

(3') $H_{A} u$ is harmonic in the interior of $D-A$.

2. Green function. The Green function associated with $P$ is defined by:

$$
G(\xi, A)=\sum_{n=0}^{\infty} P_{n}(\xi, A), \quad \xi \in \mathfrak{X}, \quad A \in \mathfrak{A},
$$

where $P_{0}(\xi, A)=\chi_{A}(\xi)$.

We remark that $G$ is the analogue of the Green function in classical potential theory.

3. Potentials. Let $f \geqq 0$; we call

$$
G f(\xi)=\int f(\eta) G(\xi, d \eta)=\sum_{n=0}^{\infty} \int f(\eta) P_{n}(\xi, d \eta)
$$


the $P$-potential of $f$ if $G f$ is $P$-superregular or equivalently if $P G f<\infty$. (This definition differs slightly from the one in [6], where for any $f \geqq 0, G f$ is called its $P$ potential.)

4. Riesz decomposition. We have

(2.3) THEOREM. Every P-superregular function $u$, bounded from below, can be represented as

$$
u=u^{\infty}+G(u-P u)
$$

where $u^{\infty}=\downarrow \lim P_{n} u$ is P-regular and in fact the greatest P-regular minorant of $u$.

This theorem represents $u$ as the sum of a $P$-regular function and a $P$-potential and corresponds in classical potential theory to the Riesz representation of a positive superharmonic function as a harmonic function plus the potential of a positive mass distribution.

Clearly, a positive $P$-superregular function $u$, bounded from below, is a $P$-potential iff $u^{\infty} \equiv 0$.

For later use we shall write down two special cases of (2.4):

$$
\begin{gathered}
1=\lim _{n \rightarrow \infty} P_{n} 1+G(1-P 1), \\
H_{A} 1=\lim _{n \rightarrow \infty} P_{n} H_{A} 1+G\left(H_{A} 1-P H_{A} 1\right) .
\end{gathered}
$$

If $P$ is given by (1.7), then clearly $\psi$, defined by (1.5), is the greatest $P$-regular minorant of 1 , i.e.,

$$
\psi=\lim _{n \rightarrow \infty} P_{n} 1
$$

We want to conclude this section with some remarks about the probabilistic side.

Given a substochastic transition function $P$ on $(\mathfrak{X}, \mathfrak{A})$, one can construct a Markov process which plays the same role in the corresponding potential theory as Brownian motion does in classical potential theory: Let $\mathfrak{X}^{\prime}=\mathfrak{X} \cup\{\boldsymbol{\rho}\}$, where $\rho$ is an adjoined element ("death state"), let $\mathfrak{X}^{\prime}$ consist of the sets of $\mathfrak{A}$ and their unions with $\{\rho\}$; we extend $P$ to a stochastic transition function $P^{\prime}$ by setting

$$
\begin{aligned}
& P^{\prime}(\xi,\{\rho\})=1-P(\xi, \mathfrak{X}), \quad \xi \in \mathfrak{X}, \\
& P^{\prime}(\rho,\{\rho\})=1 .
\end{aligned}
$$

Then, as is well known [12], for each $\xi \in \mathfrak{X}\left(\mathfrak{X}^{\prime}\right)$, there is a Markov process $\left\{X_{\xi n}, n \geqq 0\right\}$ on a probability space $\left(\Omega_{\xi}, \mathfrak{A}_{\xi}, \operatorname{Pr}_{\xi}\right)$ with state space $\mathfrak{X}^{\prime}$, stationary transition function $P^{\prime}$ and starting point $\xi$, i.e.,

(1) $\operatorname{Pr}_{\xi}\left\{X_{\xi n+1} \in A \mid X_{\xi n}\right\}=P^{\prime}\left(X_{\xi n}, A\right), \operatorname{Pr}_{\xi}$-a.e., $A \in \mathfrak{A}^{\prime}$,

(2) $\operatorname{Pr}_{\xi}\left\{X_{\xi 0}=\xi\right\}=1$.

Clearly $\operatorname{Pr}_{\xi}\left\{X_{\xi n} \in A\right\}=P_{n}(\xi, A)$ for $\xi \in \mathfrak{X}, A \in \mathfrak{A}$. We shall call the random variable $\zeta_{\xi}=\sup \left\{n ; X_{\xi n} \in \mathfrak{X}\right\}$ on $\left(\Omega_{\xi}, \mathfrak{A}_{\xi}, \operatorname{Pr}_{\xi}\right)$ the life-time of the process $\left\{X_{\xi n}, n \geqq 0\right\}$. 
For any extended real-valued function $f$ on $\mathfrak{X}$ we denote by $f^{\prime}$ its extension to $\mathfrak{X}^{\prime}$ by $f^{\prime}(\rho)=0$. Now if $u$ is $P$-superregular ( $P$-regular), then for any $\xi \in \mathfrak{X},\left\{u^{\prime}\left(X_{\xi n}\right)\right.$, $n \geqq 0\}$ is a supermartingale (martingale) with the parameter value 0 excluded in the first case if $u(\xi)=\infty$. (This is the analogue of a theorem about Brownian motion and superharmonic functions.) The supermartingale convergence theorem states:

If for a supermartingale $\left\{Y_{n}, n \geqq 0\right\}, \sup _{n} E\left|Y_{n}\right|<\infty$, then $\lim _{n \rightarrow \infty} Y_{n}$ exists and is finite a.e.

Therefore if $u$ is $P$-superregular and bounded from below, then $\lim _{n \rightarrow \infty} u^{\prime}\left(X_{\xi n}\right)$ exists and is finite $\operatorname{Pr}_{\xi}$-a.e.

In the special case when $P^{\prime}$ corresponds to $P$ of $(1.7)$ we get for the corresponding process with state space $R^{+} \cup\{\rho\}$

$$
X_{\xi n}<X_{\xi n+1} \text { or } X_{\xi n+1}=\rho, \quad \operatorname{Pr}_{\xi} \text {-a.e., }
$$

we shall see in $\$ 3$ that

$$
X_{\xi n} \rightarrow \infty \text { or } \rho, \quad \operatorname{Pr}_{\xi} \text {-a.e. }
$$

This and (2.7) imply

$$
\psi(\xi)=\operatorname{Pr}_{\xi}\left\{X_{\xi n} \rightarrow \infty\right\},
$$

since $P_{n} 1=\operatorname{Pr}_{\xi}\left\{X_{\xi n} \in R^{+}\right\}=\operatorname{Pr}_{\xi}\left\{\zeta_{\xi} \geqq n\right\}$. (2.9) explains probabilistically why we shall restrict ourselves to the case $\psi \not \equiv 0$ when studying the Martin boundary.

$P$ of (1.7) is stochastic iff $\mu(\xi, \xi+1] \equiv 1$ (or equivalently $\psi \equiv 1$ ). In this case

$$
X_{\xi n}<X_{\xi n+1}, \quad X_{\xi n} \rightarrow \infty, \quad \operatorname{Pr}_{\xi} \text {-a.e. }
$$

In $\S 6$ we shall study the relation between this process and a certain random walk.

We shall give now the probabilistic interpretation of some of the concepts given above in terms of the corresponding $X_{\xi n}$ process. We get

$$
\begin{aligned}
H_{A}^{(k)}(\xi, B) & =\operatorname{Pr}_{\xi}\left\{\tau_{\xi A}=k, X_{\xi k} \in B\right\}, \\
H_{A}(\xi, B) & =\operatorname{Pr}_{\xi}\left\{X_{\xi \tau_{\xi A}} \in B, \tau_{\xi A}<\infty\right\},
\end{aligned}
$$

where

$$
\begin{aligned}
\tau_{\xi A} & =\min \left\{n \geqq 0, X_{\xi n} \in A\right\} \quad \text { if }\left\{n \geqq 0, X_{\xi n} \in A\right\} \neq \varnothing, \\
& =\infty \text { otherwise }
\end{aligned}
$$

is the first time $X_{\xi n}$ meets $A$.

$$
\begin{aligned}
H_{A} 1(\xi) & =\operatorname{Pr}_{\xi}\left\{\tau_{\xi A}<\infty\right\}=\operatorname{Pr}_{\xi}\left\{X_{\xi n} \in A \text { for some } n \geqq 0\right\}, \\
\left(H_{A} 1\right)^{\infty}(\xi) & =\operatorname{Pr}_{\xi}\left\{X_{\xi n} \in A \text { i.o. }\right\}, \\
G\left(H_{A} 1-P H_{A} 1\right)(\xi) & =\operatorname{Pr}_{\xi}\left\{X_{\xi n} \in A \text { a finite strictly positive number of times }\right\}, \\
\left(H_{A} 1-P H_{A} 1\right)(\xi) & =0 \quad \text { if } \xi \notin A, \\
& =\operatorname{Pr}_{\xi}\left\{X_{\xi n} \notin A, n \geqq 1\right\} \quad \text { if } \xi \in A .
\end{aligned}
$$

The last four formulas give a probabilistic interpretation of (2.6). 
(b) $G(\xi, A)=E T_{\xi A}$, where $T_{\xi A}=\sum_{n=0}^{\infty} \chi_{A}\left(X_{\xi n}\right)$ is the number of entries of $X_{\xi A}$ into $A$.

We shall return now to $P$ defined by (1.7).

3. Preliminary results. We shall study first some properties of the Green function $G$. For $\xi \in R^{+}, G(\xi, \cdot)$ is a measure on the Borel subsets of $R^{+}$. We extend it to a measure on the Borel subsets of $[-1, \infty)$ by letting $G(\xi, A)=G(\xi, A \cap[0, \infty))$.

(3.1) TheOREM. For each $\xi \in R^{+}$and each Borel set $A \subseteq R^{+}$,

$$
G(\xi, A)=P_{0}(\xi, A)+\int_{A} g(\xi, \eta) \mu(d \eta)
$$

where

$$
g(\xi, \eta)=G(\xi,[\eta-1, \eta)) \text { for } \xi, \eta \in R^{+},
$$

i.e., for each $\xi \in R^{+}$, the measure $G^{\prime}(\xi, \cdot)=G(\xi, \cdot)-P_{0}(\xi, \cdot)$ on the Borel sets of $R^{+}$ is absolutely continuous with respect to $\mu$ and $g(\xi, \cdot)$ is one version of the corresponding density.

Proof. From the definition of $G$ we get

$$
\int_{R^{+}} G(\xi, d \eta) P(\eta, A)=G(\xi, A)-P_{0}(\xi, A)
$$

Substituting $P(\eta, A)=\mu\{A \cap(\eta, \eta+1]\}$ we get for the left side

$$
\int_{R^{+}} G(\xi, d \eta) \int_{A} \chi_{(\eta, \eta+1]}(\zeta) \mu(d \zeta)
$$

which by Fubini's theorem is equal to

$$
\int_{A} \mu(d \zeta) \int_{R^{+}} \chi_{[\zeta-1, \zeta)}(\eta) G(\xi, d \eta)=\int_{A} G(\xi,[\zeta-1, \zeta)) \mu(d \zeta) \text {. Q.E.D. }
$$

We have immediately from the definition of $g$

$$
\begin{array}{ll}
g(\xi, \eta) \geqq 0 & \text { for } \xi, \eta \in R^{+}, \\
g(\xi, \eta)=0 & \text { for } \xi, \eta \in R^{+}, \xi \geqq \eta .
\end{array}
$$

Therefore $g$ is not symmetrical in its two arguments as is the classical Green function. Also different from the classical situation is the boundedness of $g$, which we shall prove in the following theorem.

(3.2) Theorem. $g(\xi, \eta) \leqq 4$ for $\xi, \eta \in R^{+}$.

Since $G(\xi, A)=\chi_{A}(\xi)+\int_{A} g(\xi, \eta) \mu(d \eta)$, this theorem implies:

$$
G(\xi, A) \leqq 4 \mu(A)+1
$$


For the proof of Theorem (3.2) we need the following lemma.

(3.4) Lemma. Let $\xi_{1}, \xi_{2} \in R^{+}, \xi_{1}<\xi_{2}, \mu\left(\xi_{1}, \xi_{2}\right)<1$. Then for any $\eta_{0} \in R^{+}$: $G\left(\eta_{0},\left[\xi_{1}, \xi_{2}\right)\right) \leqq 1 /\left(1-\mu\left(\xi_{1}, \xi_{2}\right)\right)$.

Proof. Let $I=\left[\xi_{1}, \xi_{2}\right), \mu_{0}=\mu\left(\xi_{1}, \xi_{2}\right)$. For $\eta_{0} \in I$ we have

$$
P_{n}\left(\eta_{0}, I\right) \leqq \mu_{0}^{n}, \quad n \geqq 0 .
$$

This follows easily by induction from $P(\xi, A)=\mu\{(\xi, \xi+1] \cap A\}$ and from definition of the $P_{n}$ as the "iterates" of $P$.

For arbitrary $\eta_{0} \in R^{+}$we shall now prove the inequality:

$$
P_{n}\left(\eta_{0}, I\right) \leqq \sum_{k=0}^{n} \mu_{0}^{n-k} H^{(k)}\left(\eta_{0}, I\right), \quad n \geqq 0
$$

If $\eta_{0} \in I$, then $H^{(k)}\left(\eta_{0}, I\right)=1$ for $k=0,0$ for $k>0$; hence (3.6) is just (3.5).

If $\eta_{0} \notin I$, then $H^{(0)}\left(\eta_{0}, I\right)=0$, hence (3.6) is trivial for $n=0$; for $n \geqq 1$ it becomes

$$
P_{n}\left(\eta_{0}, I\right) \leqq \sum_{k=1}^{n} \mu_{0}^{n-k} H^{(k)}\left(\eta_{0}, I\right)
$$

This inequality is proved as follows:

$$
\begin{gathered}
P_{n}\left(\eta_{0}, I\right)=\int_{R^{+}} P\left(\eta_{0}, d \eta_{1}\right) \int_{R^{+}} \cdots \int_{R^{+}} P\left(\eta_{n-2}, d \eta_{n-1}\right) \int_{I} P\left(\eta_{n-1}, d \eta_{n}\right) \\
P_{n}\left(\eta_{0}, I\right)=\sum_{k=1}^{n} \int_{I^{c}} P\left(\eta_{0}, d \eta_{1}\right) \int_{I^{c}} \cdots \int_{I} P\left(\eta_{k-1}, d \eta_{k}\right) \\
\int_{R+} \cdots \int_{R+} P\left(\eta_{n-2}, d \eta_{n-1}\right) \int_{I} P\left(\eta_{n-1}, d \eta_{n}\right) \\
P_{n}\left(\eta_{0}, I\right)=\sum_{k=1}^{n} \int H^{(k)}\left(\eta_{0}, d \eta_{k}\right) P_{n-k}\left(\eta_{k}, I\right) .
\end{gathered}
$$

Substituting (3.5) in (3.8) finishes the proof of (3.6).

We remark that (3.8) appears in probabilistic notation as

$$
\operatorname{Pr}_{n_{0}}\left(X_{n_{0} n} \in I\right)=\sum_{k=1}^{n} \int_{I} \operatorname{Pr}_{\eta}\left\{X_{n n-k} \in I\right\} \operatorname{Pr}_{\eta_{0}}\left\{\tau_{\eta_{0} I}=k, X_{\eta_{0} k} \in d \eta\right\}
$$

We shall see now how (3.6) implies the lemma: For $k \geqq 0$, let $\alpha_{k}=H_{I}^{(k)}\left(\eta_{0}, I\right)$ $=\operatorname{Pr}_{\eta_{0}}\left\{\tau_{n_{0} I}=k\right\}$. Then we conclude from (3.6)

$$
\begin{aligned}
\sum_{n=0}^{N} P_{n}\left(\eta_{0}, I\right) & \leqq \sum_{n=0}^{N} \sum_{k=0}^{n} \mu_{0}^{n-k} \alpha_{k}=\sum_{k=0}^{N} \alpha_{k} \sum_{n=k}^{N} \mu_{0}^{n-k} \\
& \leqq \frac{1}{1-\mu_{0}} \sum_{k=0}^{\infty} \alpha_{k} \leqq \frac{1}{1-\mu_{0}}
\end{aligned}
$$


for all $N$ and for $\eta_{0} \in R^{+}$. We have therefore proved

$$
G\left(\eta_{0}, I\right) \leqq \sum_{n=0}^{\infty} P_{n}\left(\eta_{0}, I\right) \leqq \frac{1}{1-\mu_{0}} \text { for } \eta_{0} \in R^{+} \quad \text { Q.E.D. }
$$

Proof of Theorem (3.2). It is clear that we may assume $\eta-1 \geqq 0$. If $\mu(\eta-1, \eta)$ $\leqq \frac{1}{2}$, then Lemma (3.4) implies immediately

$$
g(\xi, \eta)=G(\xi,[\eta-1, \eta)) \leqq 2 \text { for } \xi \in R^{+} .
$$

If $\mu(\eta-1, \eta)>\frac{1}{2}$, let $\eta_{0} \in(\eta-1, \eta)$ be defined by

$$
\eta_{0}=\inf \left\{\eta^{\prime} ; \eta^{\prime} \leqq \eta, \mu\left(\eta^{\prime}, \eta\right)<\frac{1}{2}\right\}
$$

Then we have $\mu\left(\eta_{0}, \eta\right) \leqq \frac{1}{2}, \mu\left[\eta_{0}, \eta\right) \geqq \frac{1}{2}$.

From $\mu\left(\eta_{0}, \eta\right) \leqq \frac{1}{2}$, and Lemma (3.4) we conclude: $G\left(\xi,\left[\eta_{0}, \eta\right)\right) \leqq 2$ for $\xi \in R^{+}$.

From $\mu\left[\eta_{0}, \eta\right) \geqq \frac{1}{2}$ and $\mu(\eta-1, \eta) \leqq 1$ we get $\mu\left(\eta-1, \eta_{0}\right) \leqq \frac{1}{2}$, which together with Lemma (3.4) implies that $G\left(\xi,\left[\eta-1, \eta_{0}\right)\right) \leqq 2$ for $\xi \in R^{+}$.

We have therefore proved: $G(\xi,[\eta-1, \eta)) \leqq 4$ for $\xi, \eta \in R^{+}$. Q.E.D.

Following [6] a Borel set $A \subseteq R^{+}$is called transient if $G(\xi, A)<\infty$ for all $\xi \in R^{+}$. We have the following

(3.9) TheOREM. Let $A \subseteq R^{+}$be a Borel set. Then

(1) $\mu(A)<\infty$ implies that $A$ is transient. (Therefore all bounded Borel sets in $R^{+}$ are transient.)

(2) The converse is true under the assumption $\psi \neq \equiv 0$.

(1) is a trivial consequence of (3.3); we shall give the proof of (2) after (3.16) and remark here only that the converse of (1) is not true if we omit the assumption that $\psi \not \equiv 0$ : Let $\mu$ assign mass $\frac{1}{2}$ to each integer, then $G\left(\xi, R^{+}\right)=2$ for $\xi \in R^{+}$, $\mu\left(R^{+}\right)=\infty, \psi \equiv 0$.

Part (1) of Theorem (3.9) enables us to prove the following corollary about the process $\left\{X_{\xi n}\right\}$ corresponding to our substochastic transition function $P^{\prime}$.

(3.10) Corollary. For all $\xi, \operatorname{Pr}_{\xi}\left\{X_{\xi n} \rightarrow \rho\right.$ or $\left.\infty\right\}=1$.

Proof. By (3.9) we have for $\xi \in R^{+}$and any bounded Borel set $B \subseteq R^{+}, G(\xi, B)$ $<\infty$. Now

$$
G(\xi, B)=E T_{\xi B}
$$

where $T_{\xi B}$ is the number of entries of $X_{\xi n}$ into $B$. We conclude that $T_{\xi B}<\infty \operatorname{Pr}_{\xi}$-a.e. Q.E.D.

We call an extended real-valued Borel measurable function $h \geqq 0$ on $R^{+}, P$-regular on a set $A \subseteq R^{+}$if $h$ is finite on $A$, and $P h(\xi)=h(\xi)$ for $\xi \in A$. 
(3.12) THEOREM. Let $\xi<\eta, 0 \leqq h \leqq \infty$. If $h$ is $P$-regular on $[\xi, \eta]$, then

$$
h(\xi)=H_{(\eta, n+1\}} h(\xi) .
$$

Proof. We shall prove first

$$
h(\xi)=\sum_{k=0}^{n} H_{(n, \eta+1]}^{(k)} h(\xi)+\int_{[\xi, \eta]} h(\zeta) P_{n}(\xi, d \zeta), \quad n \geqq 0 .
$$

This follows immediately by induction (for $n$ ) if we prove

$$
\int_{[\xi, \eta]} h(\zeta) P_{n}(\xi, d \zeta)=H_{(\eta, \eta+1]}^{(n+1)} h(\xi)+\int_{[\xi, \eta]} h(\zeta) P_{n+1}(\xi, d \zeta), \quad n \geqq 0 .
$$

The latter formula can be obtained as follows:

$$
\begin{aligned}
\int_{[\xi, \eta]} h(\zeta) P_{n}(\xi, d \zeta) & =\int_{[\xi, \eta]}\left\{\int h\left(\zeta^{\prime}\right) P\left(\zeta, d \zeta^{\prime}\right)\right\} P_{n}(\xi, d \zeta) \\
& =\int_{[\xi, \eta]}\left\{\int_{[\xi, \eta]}+\int_{(\eta, \eta+1]}\right\} P_{n}(\xi, d \zeta) \\
& =\int_{[\xi, \eta]} h\left(\zeta^{\prime}\right) P_{n+1}\left(\xi, d \zeta^{\prime}\right)+\int_{(\eta, n+1]} h\left(\zeta^{\prime}\right) \int_{[\xi, \eta]} P_{n}(\xi, d \zeta) P\left(\zeta, d \zeta^{\prime}\right)
\end{aligned}
$$

Now (3.14) implies the lemma, since we shall see that

$$
\lim _{n \rightarrow \infty} \int_{[\xi, \eta]} h(\zeta) P_{n}(\xi, d \zeta)=0 .
$$

This equation is true because

(1) $h$ is bounded on $[\xi, \eta]$, since it is finite on $[\xi, \eta]$, is right continuous on $[\xi, \eta)$ and has finite left limits on $(\xi, \eta]$.

(2) $\lim _{n \rightarrow \infty} P_{n}(\xi,[\xi, \eta])=0$, since $[\xi, \eta]$ is transient and therefore $G(\xi,[\xi, \eta])<\infty$.

We want to add some remarks concerning the preceding proof, from the probabilistic point of view:

By (3.10) we have $\tau=\tau_{\xi,(n, \eta+1] \cup\{\rho\}}<\infty \operatorname{Pr}_{\xi}$-a.e. (3.13) is, in probabilistic notation,

$$
h(\xi)=E h^{\prime}\left(X_{\xi \tau}\right) \text {. }
$$

If we denote by $Y_{n}$ the process $X_{\xi n}$, stopped whenever it leaves $[\xi, \eta]$, i.e.,

$$
\begin{aligned}
Y_{n} & =X_{\xi n} & & \text { for } \tau>n, \\
& =X_{\xi \tau} & & \text { for } \tau \leqq n,
\end{aligned}
$$

then (3.14) is, in probabilistic notation,

$$
h(\xi)=E h^{\prime}\left(Y_{n}\right)
$$

(From this we could derive that $\left\{h^{\prime}\left(Y_{n}\right)\right\}$ is a martingale.) Now it is clear that

$$
\lim _{n \rightarrow \infty} h^{\prime}\left(Y_{n}\right)=h^{\prime}\left(X_{\xi \tau}\right), \quad \operatorname{Pr}_{\xi} \text {-a.e. }
$$


From (3.14'), (3.15) and the Fatou-Lebesgue convergence theorem we can conclude:

$$
h(\xi) \geqq E h^{\prime}\left(X_{\xi \tau}\right) .
$$

Deriving however (3.13') from (3.14') and (3.15) is equivalent to deriving uniform integrability of the $h^{\prime}\left(Y_{n}\right)$. This can be done as follows: Let $N \geqq \sup _{\zeta \in[\xi, n]} h(\zeta)$; then

$$
\left\{h^{\prime}\left(Y_{n}\right)>N\right\} \subseteq\left\{h^{\prime}\left(Y_{n}\right)=h^{\prime}\left(X_{\xi \tau}\right), h^{\prime}\left(X_{\xi \tau}\right)>N\right\}
$$

and hence

$$
\begin{aligned}
0 \leqq E\left\{h^{\prime}\left(Y_{n}\right) ; h^{\prime}\left(Y_{n}\right)>N\right\} & =E\left\{h^{\prime}\left(X_{\xi \tau}\right) ; h^{\prime}\left(Y_{n}\right)>N\right\} \\
& \leqq E\left\{h^{\prime}\left(X_{\xi \tau}\right) ; h^{\prime}\left(X_{\xi \tau}\right)>N\right\},
\end{aligned}
$$

which implies $\lim _{N \rightarrow \infty} E\left\{h^{\prime}\left(Y_{n}\right) ; h^{\prime}\left(Y_{n}\right)>N\right\}=0$ uniformly in $n$.

(3.16) THEOREM. $\psi(\xi) \leqq g(\xi, \eta)$ for $\xi<\eta-1$.

Proof. We have by (3.13): $\psi(\xi)=H_{(\eta-1, n]} \psi(\xi)$ for $\xi \leqq \eta-1$; hence $\psi(\xi)$ $\leqq H_{(\eta-1, \eta]} 1(\xi)$ for $\xi \leqq \eta-1$. From the definition of $H$ and $G$ we conclude

$$
H_{(\eta-1, \eta]} 1(\xi) \leqq G(\xi,(\eta-1, \eta]) \text { for } \xi, \eta \in R^{+},
$$

hence

$$
\psi(\xi) \leqq G(\xi,(\eta-1, \eta]) \text { for } \xi \leqq \eta-1,
$$

therefore

$$
\psi(\xi) \leqq G(\xi,[\eta-1, \eta))=g(\xi, \eta) \text { for } \xi<\eta-1 . \quad \text { Q.E.D. }
$$

The preceding proof appears even more trivial if we use probabilistic notation. We have for $\xi<\eta-1$

$$
\begin{aligned}
\psi(\xi)= & \operatorname{Pr}_{\xi}\left\{X_{\xi n} \rightarrow \infty\right\} \leqq \operatorname{Pr}_{\xi}\left(\bigcup_{n=0}^{\infty}\left\{\omega_{\xi} ; X_{\xi \eta}\left(\omega_{\xi}\right) \in[\eta-1, \eta)\right\}\right) \\
\leqq \sum_{n=0}^{\infty} \operatorname{Pr}_{\xi}\left\{\omega_{\xi} ; X_{\xi n}\left(\omega_{\xi}\right) \in[\eta-1, \eta)\right\} & =\sum_{n=0}^{\infty} P_{n}(\xi,[\eta-1, \eta)) \\
& =G(\xi,[\eta-1, \eta))=g(\xi, \eta) .
\end{aligned}
$$

Proof of Theorem (3.9) (2). The proof follows immediately from

$$
\psi(\xi) \mu\{A \cap(\xi+1, \infty)\} \leqq G(\xi, A),
$$

which follows from (3.1) and (3.16).

We shall prove now an analogue of the classical Harnack inequality for harmonic functions in the unit ball of $R^{n}$.

(3.18) TheOREM. Let $\xi<\eta, 0 \leqq h \leqq \infty$. If $h$ is $P$-regular on $[\xi, \eta]$, then

$$
h(\xi) \leqq G(\xi,[\xi, \eta]) h(\eta) .
$$


Proof. By Theorem (3.12) we have:

$$
h(\xi)=H_{(n, \eta+1\}} h(\xi)=\sum_{n=1}^{\infty} H_{(n, \eta+1\}}^{(n)} h(\xi)
$$

where

$$
H_{(\eta, n+1]}^{(n)} h(\xi)=\int_{(\eta, n+1]} h\left(\zeta^{\prime}\right) \int_{[\xi, \eta]} P_{n-1}(\xi, d \zeta) P\left(\zeta, d \zeta^{\prime}\right)
$$

Since for $\zeta \in[\xi, \eta]$ and any Borel set $A$ of $(\eta, \eta+1], P(\zeta, A) \leqq P(\eta, A)=\mu(A)$, we get $H_{(\eta, \eta+1]}^{(n)} h(\xi) \leqq h(\eta) P_{n-1}(\xi,[\xi, \eta])$ which implies the theorem.

(3.19) CoRollary. If $h \geqq 0$ is P-regular and $\lim \inf _{\xi \rightarrow \infty} h(\xi)=0$ then $h \equiv 0$.

Proof. Let $\xi_{n} \uparrow \infty$ be such that $\lim _{n \rightarrow \infty} h\left(\xi_{n}\right)=0$. By Theorem (3.12) we get

$$
h(\xi)=H_{\left(\xi_{n}-1, \xi_{n}\right]} h(\xi) \text { for } \xi \leqq \xi_{n}-1 .
$$

By the Harnack inequality (3.18) and the upper estimate for $g$ (3.2) we conclude

$$
h(\eta) \leqq 4 h\left(\xi_{n}\right) \text { for } \eta \in\left(\xi_{n}-1, \xi_{n}\right]
$$

hence $h(\xi) \leqq 4 h\left(\xi_{n}\right) H_{\left(\xi_{n}-1, \xi_{n}\right]} 1(\xi) \leqq 4 h\left(\xi_{n}\right)$ for $\xi \leqq \xi_{n}-1$, which implies the corollary.

We shall conclude this section with a remark about the set of zeros of a $P$-regular function $h \geqq 0$ (e.g., $\psi$ ). From (3.18) and (3.9) (1) we see that $h\left(\xi_{0}\right)=0$ implies $h(\xi)=0$ for $\xi \leqq \xi_{0}$. Hence, if $h \not \equiv 0$ the set of zeros of $h$ is either empty or an interval $[0, a)$ or $[0, a] ; h(a)=0$ iff $\mu(a, a+1]=0$.

4. Basic results. The Riesz decomposition theorem (2.3) gives the decomposition of a $P$-superregular function, bounded from below, into the sum of a $P$-potential and a $P$-regular function. We shall determine now the class of $P$-regular functions, bounded from below, in the case $\psi \not \equiv 0$.

(4.1) THEOREM. If $\psi \not \equiv 0$, any P-regular function $h$, bounded from below (or from above), is proportional to $\psi$.

Proof. (1) It is sufficient to prove the theorem for nonnegative $P$-regular functions: If $h$ is $P$-regular and bounded from below, say $h \geqq-\alpha(\alpha>0)$, then $h_{1}=h+\alpha \psi$ $=\lim _{n \rightarrow \infty} P_{n}(h+\alpha)$ is $P$-regular and $\geqq 0$; if $h$ is $P$-regular and bounded from above, consider $-h$.

(2) So let $h \geqq 0$ be $P$-regular. We shall prove that $\psi \not \equiv 0$ implies that $h$ is bounded. Assume $h$ is not bounded and let $\xi_{n} \rightarrow \infty$ be such that $\lim _{n \rightarrow \infty} h\left(\xi_{n}\right)=\infty$. By the Harnack inequality (3.18) we get

$$
h\left(\xi_{n}\right) \leqq G\left(\xi_{n},\left[\xi_{n}, \eta\right]\right) h(\eta) \text { for } \xi_{n}<\eta .
$$

This together with (3.2) implies

$$
h\left(\xi_{n}\right) \leqq 5 h(\eta) \text { for } \eta \in\left(\xi_{n}, \xi_{n}+1\right]
$$


Since by Theorem (3.12)

$$
h(\xi)=H_{\left(\xi_{n}, \xi_{n}+1\right]} h(\xi) \text { for } \xi \leqq \xi_{n},
$$

we get

$$
h(\xi) \geqq \frac{1}{5} h\left(\xi_{n}\right) H_{\left(\xi_{n}, \xi_{n}+1\right]} 1(\xi) \text { for } \xi \leqq \xi_{n},
$$

hence

$$
h(\xi) \geqq \frac{1}{5} h\left(\xi_{n}\right) \psi(\xi) \text { for } \xi \leqq \xi_{n} .
$$

This implies that $h(\xi) \equiv \infty$ for all $\xi \in R^{+}$for which $\psi(\xi)>0$, which contradicts the finiteness of $h$.

(3) So let $a=\sup _{\xi \in R^{+}} h(\xi)$; we just proved that $a<\infty$ and shall prove now that $h=a \psi$. Assume $a>0$. We shall see first that $\lim \sup _{\xi \rightarrow \infty} h(\xi)=a$. If this were not true, there would be an $\varepsilon>0$ and a $\xi_{1} \in R^{+}$such that $h(\xi) \leqq a-\varepsilon$ for $\xi \geqq \xi_{1}$, and therefore we would get by Theorem (3.12)

$$
h(\xi)=H_{\left(\xi_{1}, \xi_{1}+1\right]} h(\xi) \leqq(a-\varepsilon) H_{\left(\xi_{1}, \xi_{1}+1\right]} 1(\xi) \leqq a-\varepsilon \text { for } \xi \leqq \xi_{1},
$$

and hence $h \leqq a-\varepsilon$, which contradicts $\sup _{\xi \in R^{+}} h(\xi)=a$. Now let $h_{1}$ $=\lim _{n \rightarrow \infty} P_{n}(a-h)=a \psi-h$; then $h_{1} \geqq 0$ is $P$-regular and $\lim _{\inf _{\xi \rightarrow \infty}} h_{1}(\xi)=0$; hence we have by Corollary (3.19) $h_{1} \equiv 0$, i.e., $h=a \psi$.

REMARK. In general the class of all $P$-regular functions is of course much larger than the class of multiples of $\psi$. For example, let $\mu$ be the Lebesgue measure on $R^{+}$. Then $\psi \equiv 1$, and for any $C^{\infty}$ function $h_{0}$ on $(0,1)$ with compact support there is a $C^{\infty}$ function $h$ on $R^{+}$which coincides with $h_{0}$ on $(0,1)$ and is $P$-regular, or equivalently satisfies

$$
h(\xi+1)=h^{\prime}(\xi)+h(\xi) .
$$

REMARK. The following considerations show that we gain essentially nothing new if we consider wide-sense $P$-regular functions, i.e., extended real-valued (rather than real-valued) functions $h$ on $R^{+}$, satisfying the integral equation $h(\xi)$ $=\int_{(\xi, \xi+1]} h(\eta) \mu(d \eta)$. By the same argument as in the proof of Theorem (4.1) it is possible to restrict the study of wide-sense $P$-regular functions, bounded from below, to the study of nonnegative wide-sense $P$-regular functions. We shall see that the class of nonnegative wide-sense $P$-regular functions can be completely determined by an analysis of the sets of infinities of the functions in this class. We shall now give this analysis. We shall verify first:

(1) If a wide-sense $P$-regular function $h \geqq 0$ is finite at $\xi_{1}$ and $\xi_{2}$, where $\left|\xi_{1}-\xi_{2}\right|$ $\leqq 1$, then $h$ is finite on $\left[\xi_{1}, \xi_{2}\right]$.

(2) If a wide-sense $P$-regular function $h \geqq 0$ is finite on an interval $(\xi, \xi+\varepsilon]$, then $h$ is finite at $\xi$.

The proof of (1) follows immediately from the defining integral equation. (2) is proved as follows: Since $h$ is $P$-regular on $(\xi, \xi+\varepsilon]$ we conclude by (3.18)

$$
h\left(\xi^{\prime}\right) \leqq G(\xi ;[\xi, \xi+\varepsilon]) h(\xi+\varepsilon) \text { for } \xi^{\prime} \in(\xi, \xi+\varepsilon] .
$$


We may of course assume that $\varepsilon<1$; by (3.2) we get then

$$
h\left(\xi^{\prime}\right) \leqq 4 h(\xi+\varepsilon) \text { for } \xi^{\prime} \in(\xi, \xi+\varepsilon]
$$

which implies $h(\xi)<\infty$.

From (1), (2) and the defining integral equation we conclude:

(A) For every wide-sense $P$-regular function $h>0$, the set of infinities of $h$ is the union of a finite or infinite number of (nondegenerate) disjoint intervals $I_{n}$ with the following properties $\left(\xi_{n}\right.$ and $\xi_{n}^{\prime}(\leqq \infty)$ denote the left and right endpoints of $\left.I_{n}\right)$ :

(a) $I_{n}$ is open on the right,

(b) $\mu\left\{(\xi, \xi+1] \cap I_{n}\right\}>0$ for $\xi \in I_{n}$,

(c) either $\mu\left(\xi_{n}, \xi_{n}+1\right]=0$ or $\mu(\xi, \xi+1]=0$ if $\xi \in\left(\xi_{n}-\delta, \xi_{n}\right)$ for some $\delta>0$,

(d) $\xi_{n} \notin I$ iff $\mu\left(\xi_{n}, \xi_{n}+1\right]=0$.

(These properties clearly imply that $\xi_{n}^{\prime}-\xi_{n}>1$ and that the $I_{n}$ are the components of $\bigcup I_{n}$.)

(B) Conversely given any finite or infinite number of disjoint intervals $I_{n}$ with the properties (a)-(d), we can define a wide-sense $P$-regular function $h \geqq 0$ with $\bigcup I_{n}$ as its set of infinities:

If the set $\bigcup I_{n}$ is unbounded, let

$$
\begin{aligned}
h & =\infty \text { on } \bigcup I_{n}, \\
& =0 \text { off } \bigcup I_{n} .
\end{aligned}
$$

If the set $\bigcup I_{n}$ is bounded, denote by $\xi^{*}$ the right endpoint of the last $I_{n}\left(\xi^{*}=0\right.$, if $\left.\bigcup_{n} I_{n}=\varnothing\right)$ and let

$$
\begin{aligned}
h(\xi) & =\infty & & \text { on } \bigcup I_{n}, \\
& =h_{1}(\xi) & & \text { for } \xi \geqq \xi^{*}, \\
& =0 & & \text { otherwise, }
\end{aligned}
$$

where $h_{1}$ is any $P$-regular function on $\left[\xi^{*}, \infty\right)$. It is also clear that there can be no other wide-sense $P$-regular function which has $\bigcup I_{n}$ as its set of infinities.

We shall now state two theorems which will follow from the Riesz decomposition theorem (2.3) and the estimates (3.2) and (3.16) for the Green density $g(\xi, \eta)$.

(4.2) THEOREM. $\psi \not \equiv 0$ iff
(A)
(i) $\limsup _{\xi \rightarrow \infty} \mu(\xi, \xi+1]>0$,
(ii) $\int_{(0, \infty)}\{1-\mu(\xi, \xi+1]\} \mu(d \xi)<\infty$.

(4.3) Theorem. If $\psi \not \equiv 0$ and $v$ is a P-potential then $\lim _{\xi \rightarrow \infty} P v(\xi)=0$.

Proof of Theorems (4.2) and (4.3). Let $v$ be any $P$-potential. The Riesz decomposition formula (2.4) gives, since $v^{\infty} \equiv 0$ :

$$
v(\xi)=\int(v-P v)(\eta) G(\xi, d \eta)=(v-P v)(\xi)+\int(v-P v)(\eta) g(\xi, \eta) \mu(d \eta),
$$

hence

$$
P v(\xi)=\int_{(\xi, \infty)}(v-P v)(\eta) g(\xi, \eta) \mu(d \eta)
$$


Applying (3.2) and (3.16) to (4.4) we get

$$
\psi(\xi) \int_{(\xi+1, \infty)}(v-P v)(\eta) \mu(d \eta) \leqq P v(\xi) \leqq 4 \int_{(\xi, \infty)}(v-P v)(\eta) \mu(d \eta) .
$$

Since $P v(\xi)=\int_{(\xi, \xi+1]} v(\eta) \mu(d \eta)<\infty$, the first inequality of (4.5) implies

(4.6) If $\psi \not \equiv 0$ and $v$ a $P$-potential, then $\int_{(0, \infty)}(v-P v)(\eta) \mu(d \eta)<\infty$.

(4.6) and the second inequality of (4.5) imply Theorem (4.3). Applying (4.6) to the $P$-potential $v=1-\psi$, we see that $\psi \not \equiv 0$ implies (ii). That $\psi \not \equiv 0$ implies (i), follows from (3.19). Thus if $\psi \not \equiv 0$, (i) and (ii) are true. Conversely applying the second inequality of (4.5) to the $P$-potential $v=1-\psi$, we get:

$$
0<\mu(\xi, \xi+1]-\psi(\xi) \leqq 4 \int_{(\xi, \infty)}\{1-\mu(\eta, \eta+1]\} \mu(d \eta) .
$$

Therefore (ii) implies $\lim _{\xi \rightarrow \infty}\{\mu(\xi, \xi+1]-\psi(\xi)\}=0$; hence (i) and (ii) imply $\psi \not \equiv 0$. Q.E.D.

Since $\psi(\xi) \leqq \mu(\xi, \xi+1] \leqq 1$, it will be a consequence of Theorem (4.14) that we can replace condition (i) in Theorem (4.2) by

(i') $\lim _{\xi \rightarrow \infty} \mu(\xi, \xi+1]=1$.

We shall prove the following corollary of Theorem (4.2):

(4.7) Corollary. Let $\mu(d \xi)=K(\xi) d \xi, K \leqq 1$. Then $\psi \not \equiv 0$ iff $\int_{0}^{\infty}\{1-K(\xi)\} d \xi<\infty$.

(This necessary and sufficient condition for $\psi \not \equiv 0$ was derived by Slater and Wilf [16] for $\mu$ in this special form under the additional assumption that $K$ be nondecreasing; see §1.)

Proof. By Theorem (4.2) as amended in the light of Theorem (4.14) (to be proved below) we have $\psi \not \equiv 0$ iff

(i') $\lim _{\xi \rightarrow \infty} \int_{\xi}^{\xi+1} K(\eta) d \eta=1$,

(ii) $\int_{0}^{\infty}\left\{1-\int_{\xi}^{\xi+1} K(\eta) d \eta\right\} K(\xi) d \xi<\infty$.

The integral in (ii) is equal to $\int_{0}^{\infty} \int_{\xi}^{\xi+1}\{1-K(\eta)\} K(\xi) d \eta d \xi$, and therefore by Fubini's theorem finite iff

$$
\int_{0}^{\infty}\{1-K(\eta)\}\left(\int_{\eta-1}^{\eta} K(\xi) d \xi\right) d \eta<\infty
$$

Therefore (i') and (ii) are equivalent to $\int_{0}^{\infty}\{1-K(\eta)\} d \eta<\infty$. Q.E.D.

We shall prove another corollary of Theorem (4.2). Let $A \subseteq R^{+}$be any Borel set. Let $\mu_{A}$ be the restriction of $\mu$ to $A$, i.e., for any Borel set $B \subseteq R^{+}$, let $\mu_{A}(B)$ $=\mu(A \cap B)$; let $P_{A}, \psi_{A}$ be the $P, \psi$ corresponding to $\mu_{A}$, i.e.,

$$
\begin{aligned}
P_{A}(\xi, B) & =\mu_{A}\{(\xi, \xi+1] \cap B\}=\mu\{(\xi, \xi+1] \cap A B\} \\
\psi_{A} & =\lim _{n \rightarrow \infty}\left(P_{A}\right)_{n} 1 .
\end{aligned}
$$

Then we have

(4.8) CoRollary. If $\psi \not \equiv 0$, then $\psi_{A} \not \equiv 0$ iff $\mu\left(A^{c}\right)<\infty$. 
Proof. By Theorem (4.2), as amended in the light of Theorem (4.14) we have $\psi_{A} \not \equiv 0$ iff

(i') $\lim _{\xi \rightarrow \infty} \mu_{A}(\xi, \xi+1]=1$ and

(ii) $\int\left\{1-\mu_{A}(\xi, \xi+1]\right\} \mu_{A}(d \xi)<\infty$.

Now the integral in (ii) is equal to

$$
\int\{1-\mu(\xi, \xi+1]\} \mu_{A}(d \xi)+\int \mu_{A^{c}}(\xi, \xi+1] \mu_{A}(d \xi) .
$$

The first integral is majorized by $\int\{1-\mu(\xi, \xi+1]\} \mu(d \xi)$, which is finite, by Theorem (4.2), since $\psi \not \equiv 0$. Therefore (ii) is equivalent to

$$
\int \mu_{A^{c}}(\xi, \xi+1] \mu_{A}(d \xi)=\iint_{(\xi, \xi+1]} \mu_{A^{c}}(d \eta) \mu_{A}(d \xi)<\infty .
$$

By Fubini's theorem this holds iff

$$
\int_{(1, \infty)} \int_{[\eta-1, \eta)} \mu_{A}(d \xi) \mu_{A^{c}}(d \eta)=\int_{(1, \infty)} \mu_{A}[\eta-1, \eta) \mu_{A^{c}}(d \eta)<\infty
$$

Therefore if $\psi \not \equiv 0,\left(i^{\prime}\right)$ and (ii) are equivalent to

$$
\int \mu_{A^{c}}(d \eta)=\mu\left(A^{c}\right)<\infty .
$$

REMARK. We can interpret $\psi_{A}$ probabilistically in terms of $X_{\xi n}$. If we denote by ${ }_{A} X_{\xi n}$ the process obtained by stopping $X_{\xi n}$ whenever it reaches $A^{c}$, then $\psi_{A}(\xi)$ $=\operatorname{Pr}_{\xi}\left\{{ }_{A} X_{\xi n} \rightarrow \infty\right\}$. The probabilistic significance for the $X_{\xi n}$ process of the sets of finite $\mu$-measure will be discussed in $\$ 5$.

Theorem (4.3) implies the following corollary which is the analogue of a classical theorem.

(4.9) Corollary. Let $u \geqq 0$ be P-superregular. Then

$$
u^{\infty}=\lim _{a \rightarrow \infty} H_{(a, \infty)} u=\lim _{a \rightarrow \infty} H_{(a, a+1]} u .
$$

If $u \equiv 1$, we get in particular

$$
\psi(\xi)=\lim _{a \rightarrow \infty} H_{(a, \infty)} 1(\xi)=\lim _{a \rightarrow \infty} H_{(a, a+1]} 1(\xi) .
$$

Proof. It is clear that the expressions in the middle and on the right coincide. Because of the Riesz decomposition theorem and Theorem (3.12) it is enough to prove that for any $P$-potential $v, \lim _{a \rightarrow \infty} H_{(a, \infty)} v(\xi) \equiv 0$. We shall first prove that $H_{(a, \infty)} v=H_{(a, a+1]} v$ is finite on $[0, a]$. As in the proof of (3.18) we get

$$
\begin{aligned}
H_{(a, \infty)} v(\xi) & \leqq G(\xi,[\xi, a]) \int_{(a, a+1]} v(\eta) \mu(d \eta) \\
& =G(\xi,[\xi, a]) P v(a)<\infty \text { for } \xi \in[0, a] .
\end{aligned}
$$


This implies, since $P H_{(a, \infty)} v(\xi)=H_{(a, \infty)} v(\xi)$ for $\xi \in[0, a]$, that $H_{(a, \infty)} v$ is $P$-regular on $[0, a]$. Now we conclude, by Theorem (3.12),

$$
H_{(a, \infty)} v(\xi)=H_{(a-1, a]}\left(H_{(a, \infty)} v\right)(\xi) \text { for } \xi<a-1 .
$$

This implies

$$
H_{(a, \infty)} v(\xi) \leqq 4 P v(a) \text { for } \xi<a-1,
$$

since by (4.11) and (3.2)

$$
H_{(a, \infty)} v(\xi) \leqq 4 P v(a) \text { for } \xi \in(a-1, a] .
$$

(4.12) and (4.3) imply the corollary. Theorem (4.3) also implies

(4.13) THEOREM. If $\psi \not \equiv 0$ and $v$ is a P-potential, then there is a Borel set $A \subseteq R^{+}$, such that $\mu(A)<\infty$ and $\lim _{\xi \rightarrow \infty ; \xi \notin A} v(\xi)=0$.

Proof. We shall prove first that for any $\varepsilon>0$, the set $A_{\varepsilon}=\left\{\eta ; \eta \in R^{+}, v(\eta) \geqq \varepsilon\right\}$ has finite $\mu$-measure. Let $A_{\varepsilon}^{\prime}=\left\{\eta ; \eta \in R^{+} ; P v(\eta) \geqq \varepsilon\right\}$ and $A_{\varepsilon}^{\prime \prime}=\left\{\eta ; \eta \in R^{+} ;(v-P v)(\eta) \geqq \varepsilon\right\}$. Clearly, $A_{\varepsilon} \subseteq A_{\varepsilon / 2}^{\prime} \cup A_{\varepsilon / 2}^{\prime \prime}$.

From Theorem (4.3) we conclude that $\mu\left(A_{\varepsilon}^{\prime}\right)<\infty$ for any $\varepsilon>0$. That also $\mu\left(A_{\varepsilon}^{\prime \prime}\right)$ $<\infty$ for any $\varepsilon>0$ follows from (4.6) since $\varepsilon \mu\left(A_{\varepsilon}^{\prime \prime}\right) \leqq \int_{(0, \infty)}(v-P v)(\eta) \mu(d \eta)$. We therefore conclude that $\mu\left(A_{\varepsilon}\right)<\infty$ for any $\varepsilon>0$. To complete the proof of the theorem, choose $\xi_{n} \uparrow \infty$ such that $\mu\left\{A_{1 / n} \cap\left(\xi_{n}, \infty\right)\right\} \leqq 1 / n^{2}$ and let

$$
A=\bigcup_{n=1}^{\infty}\left\{A_{1 / n} \cap\left(\xi_{n}, \infty\right)\right\}
$$

Then $\mu(A)<\infty$ and $v(\xi)<1 / n$ for $\xi \in A^{c} \cap\left(\xi_{n}, \infty\right)$, i.e. $\lim _{\xi \rightarrow \infty ; \xi \notin A} v(\xi)=0$.

ReMARK. In general for a $P$-potential $v, \lim _{\xi \rightarrow \infty} v(\xi)$ need not exist. We shall see in $\S 5$, that $H_{A_{1}} 1(\xi)$ is a $P$-potential if $\mu\left(A_{1}\right)<\infty$. Since $H_{A_{1}} 1(\xi)=1$ for $\xi \in A_{1}$ and since $\lim _{\xi \rightarrow \infty ; \xi \notin A} H_{A_{1}} 1(\xi)=0$ for some $A$ such that $\mu(A)<\infty$ (by Theorem (4.13)), we see that $\lim _{\xi \rightarrow \infty} H_{A_{1}} 1(\xi)$ does not exist, if $A_{1}$ is unbounded.

As a consequence of Theorem (4.13) we shall prove:

(4.14) THEOREM. If $\psi \not \equiv 0$, then $\lim _{\xi \rightarrow \infty} \psi(\xi)=1$.

Proof. Since $1-\psi$ is a $P$-potential, Theorem (4.13) gives the existence of a Borel set $A \subseteq R^{+}$such that $\mu(A)<\infty$ and $\lim _{\xi \rightarrow \infty ; \xi \notin A}(1-\psi)(\xi)=0$, i.e., $\lim _{\xi \rightarrow \infty ; \xi \notin A} \psi(\xi)=1$.

We shall see now that in the case of $\psi$ this even implies:

$$
\lim _{\xi \rightarrow \infty} \psi(\xi)=1 \text {. }
$$

In order to prove $(\mathrm{X})$, we only have to show: If $(\mathrm{X})$ were not true, i.e., if for some $a \in(0,1)$ and $\xi_{n} \uparrow \infty, \psi\left(\xi_{n}\right) \leqq a$, then for any $a^{\prime} \in(a, 1)$, the set $A_{0}=\left\{\xi ; \xi \in R^{+}\right.$, $\left.\psi(\xi) \leqq a^{\prime}\right\}$ would have infinite $\mu$-measure.

This is proved as follows: Since $\psi(\xi) \leqq \mu(\xi, \xi+1]$, the assumption $\psi \not \equiv 0$ and Corollary (3.19) imply $\lim \inf _{\xi \rightarrow \infty} \mu(\xi, \xi+1]>0$. Let $\varepsilon \in(0,1)$ be such that $\varepsilon$ 
$<\lim _{n \rightarrow \infty} \mu\left(\xi_{n}-1, \xi_{n}\right]$ and $1 /(1-\varepsilon) \leqq a^{\prime} \mid a$. The first condition implies that, disregarding initial terms,

$$
\mu\left(\xi_{n}-1, \xi_{n}\right]>\varepsilon .
$$

Moreover, since $\psi$ is right continuous, we may assume that $\mu\left\{\xi_{n}\right\}=0$. Therefore we can define $l_{n} \in(0,1)$ by

$$
l_{n}=\inf \left\{l ; \varepsilon \leqq \mu\left(\xi_{n}-l, \xi_{n}\right]\right\} .
$$

Now, (1) $\mu\left(\xi_{n}-l_{n}, \xi_{n}\right] \leqq \varepsilon$, (2) $\mu\left[\xi_{n}-l_{n}, \xi_{n}\right] \geqq \varepsilon$. If we let $A_{0}^{\prime}=\bigcup\left[\xi_{n}-l_{n}, \xi_{n}\right]$, this union contains infinitely many disjoint intervals $\left[\xi_{n}-l_{n}, \xi_{n}\right]$ and by (2) we conclude that $\mu\left(A_{0}^{\prime}\right)=\infty$.

We shall see now that $A_{0}^{\prime} \subseteq A_{0}$, i.e., $\psi(\xi) \leqq a^{\prime}$ for $\xi \in A_{0}^{\prime}$. Let $\xi_{0} \in\left[\xi_{n}-l_{n}, \xi_{n}\right)$. Then we have by the Harnack inequality $(3.18) \psi\left(\xi_{0}\right) \leqq G\left(\xi_{0},\left[\xi_{0}, \xi_{n}\right]\right) \psi\left(\xi_{n}\right)$. Since $\psi\left(\xi_{n}\right) \leqq a$ and

$$
G\left(\xi_{0},\left[\xi_{0}, \xi_{n}\right]\right)=\sum_{k=0}^{\infty} P_{k}\left(\xi_{0},\left[\xi_{0}, \xi_{n}\right]\right) \leqq \sum_{k=0}^{\infty}\left\{\mu\left(\xi_{0}, \xi_{n}\right]\right\}^{k} \leqq \frac{1}{1-\varepsilon}
$$

this implies $\psi\left(\xi_{0}\right) \leqq a /(1-\varepsilon) \leqq a^{\prime}$. Q.E.D.

We are now in a position to give the

Proof of Theorem (1.6). (a) Uniqueness: If $h$ is a solution of (1.2) and (1.3), then $h$ is bounded and $\not \equiv 0$. We show first that this implies $\psi \neq \equiv 0:$ If $h \leqq a$, then $h=P_{n} h \leqq P_{n} a=a P_{n} 1$, hence $h \leqq a \psi$; if also $h\left(\xi_{0}\right)>0$, then $0<h\left(\xi_{0}\right) \leqq a \psi\left(\xi_{0}\right)$, hence $\psi \not \equiv 0$. Now we get by (4.1) $h=\alpha \psi$ and by (4.14) $\alpha=1$, hence $h=\psi$.

(b) Existence: by Theorems (4.2) and (4.14).

(4.15) CoRollaRY. If $\psi \not \equiv$, then for any P-superregular function $u \geqq 0$ (or bounded from below), $\lim _{\xi \rightarrow \infty} P u(\xi)$ exists and is finite.

(4.16) COROLlaRY. If $\psi \neq 0$, then for any P-superregular function $u \geqq 0$ (or bounded from below), there exists a Borel set $A \subseteq R^{+}$such that $\mu(A)<\infty$ and $\lim _{\xi \rightarrow \infty: \xi \notin A} u(\xi)$ exists and is finite.

(The limits in both corollaries are of course the same for each $u$.) We also have a limit theorem for $P$-regular functions if $\psi \equiv 0$.

(4.17) THEOREM. If $\psi \equiv 0$ and if $h \geqq 0$ is $P$-regular, then either $h \equiv 0$ or $\lim _{\xi \rightarrow \infty} h(\xi)$ $=\infty$.

Proof. Suppose, there are $\xi_{n} \uparrow \infty$ and $a>0$ such that $h\left(\xi_{n}\right) \leqq a$. In the proof of Corollary (3.19) we derived the formula:

$$
h(\xi) \leqq 4 h\left(\xi_{n}\right) H_{\left(\xi_{n}, \xi_{n}+1\right]} 1(\xi) \text { for } \xi \leqq \xi_{n} .
$$

By (4.10) we conclude $h \equiv 0$. Q.E.D.

5. Martin boundary. In this section we shall assume $\psi \neq \equiv 0$ or equivalently (A). We shall see that the results of the preceding section (characterization of nonnegative $P$-regular functions, limit theorems for $P$-superregular functions) can be put into the framework of a Martin boundary theory. 
We shall start by recalling for classical potential theory the concept of the Martin boundary [13]: If the open connected set $D \subseteq R^{n}$ has a Green function $g_{D}(\xi, \eta)$, $(\xi, \eta \in D)$, fix $\xi_{0} \in D$ and let $k_{D}(\eta, \xi)=g_{D}(\xi, \eta) / g_{D}\left(\xi_{0}, \eta\right)$ be the normalized Green function. The Martin space $\hat{D}$ of $D$ is roughly speaking the strongest compactification of $D$ such that $\lim _{n \rightarrow \eta_{0}: \eta \in D} k_{D}(\eta, \xi)$ exists for $\eta_{0} \in \partial D=\hat{D}-D ; \partial D$ is called the Martin boundary of $D$. (For "smooth" regions it coincides with the Euclidean boundary.) In this sense the following theorem identifies the one-point-compactification of $R^{+}$as the Martin space corresponding to our $P$-potential theory, and the set consisting of the Alexandroff point $\infty$ as Martin boundary.

(5.1) TheOREM. Let $\psi\left(\xi_{0}\right)>0$, and let $k(\eta, \xi)=g(\xi, \eta) / g\left(\xi_{0}, \eta\right)$. Then

$$
\lim _{\eta \rightarrow \infty} k(\eta, \xi)=\frac{\psi(\xi)}{\psi\left(\xi_{0}\right)}
$$

Proof. We first remark that (3.2) and (3.16) imply

$$
k(\eta, \xi) \leqq 4 / \psi\left(\xi_{0}\right) \text { for } \xi_{0}<\eta-1 \text {. }
$$

Denote now by $S(\xi)$ the set of cluster values of $k(\eta, \xi)$ as $\eta \rightarrow \infty$.

Each $S(\xi)$ contains only finite values. We have to show that $S(\xi)=\left\{\psi(\xi) / \psi\left(\xi_{0}\right)\right\}$ for each $\xi$. So let $a \in S\left(\xi_{1}\right)$ and let $\eta_{n} \rightarrow \infty$ be such that $\lim _{n \rightarrow \infty} k\left(\eta_{n}, \xi_{1}\right)=a$. The proof that $a=\psi\left(\xi_{1}\right) / \psi\left(\xi_{0}\right)$ proceeds in the following four steps:

(1) Let $C$ be a countable dense subset of $R^{+}$. From (5.2) and a diagonal argument we see that there exists a subsequence $\left\{\eta_{n}^{\prime}\right\}$ of $\left\{\eta_{n}\right\}$ such that $\lim _{n \rightarrow \infty} k\left(\eta_{n}^{\prime}, \xi\right)$ exists for $\xi \in C$. We call the limit function $a(\xi)$ and write from now on $\left\{\eta_{n}\right\}$ instead of $\left\{\eta_{n}^{\prime}\right\}$.

(2) We want to show that $\lim _{n \rightarrow \infty} k\left(\eta_{n}, \xi\right)$ exists (finite) on all $R^{+}$. From (X) and the fact that the $k\left(\eta_{n}, \cdot\right)$ are $P$-regular for $\xi<\eta_{n}-1$, i.e.,

$$
k\left(\eta_{n}, \xi\right)=\int_{(\xi, \xi+1]} k\left(\eta_{n}, \xi^{\prime}\right) \mu\left(d \xi^{\prime}\right) \text { for } \xi<\eta_{n}-1,
$$

it follows that at each $\xi^{0} \in R^{+}$the $k\left(\eta_{n}, \cdot\right)$ are uniformly right continuous, disregarding initial values of $n$. Now let $\xi^{0} \in C^{c}$. Given $\varepsilon>0$, there is a $\delta>0$ such that for sufficiently large $n$ and $\xi \in\left(\xi^{0}, \xi^{0}+\delta\right)$ :

$$
\left|k\left(\eta_{n}, \xi\right)-k\left(\eta_{n}, \xi^{0}\right)\right|<\varepsilon .
$$

Thus for $\xi \in\left(\xi^{0}, \xi^{0}+\delta\right) \cap C$

$$
a(\xi)-\varepsilon \leqq \liminf _{n \rightarrow \infty} k\left(\eta_{n}, \xi^{0}\right) \leqq \limsup _{n \rightarrow \infty} k\left(\eta_{\underline{n}}, \xi^{0}\right) \leqq a(\xi)+\varepsilon .
$$

Therefore

$$
\begin{aligned}
\limsup _{\xi \downarrow \xi^{0}: \xi \in C} a(\xi)-\varepsilon & \leqq \liminf _{n \rightarrow \infty} k\left(\eta_{n}, \xi^{0}\right) \leqq \limsup _{n \rightarrow \infty} k\left(\eta_{n}, \xi^{0}\right) \\
& \leqq \liminf _{\xi \downarrow \xi^{0}: \xi \in C} a(\xi)+\varepsilon .
\end{aligned}
$$

Hence $\lim _{n \rightarrow \infty} k\left(\eta_{n}, \xi^{0}\right)$ and $\lim _{\xi \downarrow \xi^{0} ; \xi \in C} a(\xi)$ exist and are equal. 
(3) In order to show that $a(\xi)=\lim _{n \rightarrow \infty} k\left(\eta_{n}, \xi\right)$ (defined now on $R^{+}$) is $P$-regular on $R^{+}$, we only remark that the $k\left(\eta_{n}, \cdot\right)$ are $P$-regular on $\left[0, \eta_{n}-1\right)$ and that we can apply the bounded convergence theorem for integrals because of (5.2).

(4) Now we can conclude by Theorem (4.1) that $a(\xi)=\alpha \psi(\xi)$. Since $k\left(\eta, \xi_{0}\right)$ $\equiv 1$ implies $a\left(\xi_{0}\right)=1$, we get $a(\xi)=\psi(\xi) / \psi\left(\xi_{0}\right)$, and therefore $a=a\left(\xi_{1}\right)=\psi\left(\xi_{1}\right) / \psi\left(\xi_{0}\right)$. Q.E.D.

As we mentioned in the Introduction, in classical potential theory the Martin boundary of a region $D$ (with Green function!) is closely connected with the representation of positive harmonic functions on $D$ on one hand and with the boundary behavior of positive superharmonic functions on the other. The representation theorem states that every positive harmonic function is a unique linear combination of the so-called minimal harmonic functions. The latter ones are in 1-1 correspondence with the Martin boundary points-disregarding a certain negligible set. The correspondence is given by: $\eta_{0} \rightarrow \lim _{\eta \rightarrow \eta_{0}: \eta \in D} k_{D}(\eta, \cdot)$. About the boundary behavior of positive superharmonic functions the Fatou theorems give information. One version states that any positive superharmonic function $u$ on $D$ has finite limits at almost all (with respect to "harmonic measure") boundary points along their fine neighborhood filters. This is the modern version of the classical Fatou theorems for positive harmonic and superharmonic functions on a ball in $R^{n}$, where the approach to the boundary is nontangential and radial respectively.

Now back to our $P$-potential theory! In view of Theorem (5.1) it is clear that Theorem (4.1) is the analogue of the classical representation theorem and that we may consider $\psi$ as the minimal function corresponding to the Martin boundary point $\infty$.

We shall discuss now the limit theorems of $\S 4$ for $P$-superregular and $P$-regular functions in the light of the Fatou theorems of classical potential theory.

We shall start with Corollary (4.16). We remark that the neighborhood filter of $\infty$ in the topology of the Martin space is the ordinary filter of " $\xi \rightarrow \infty$." We shall introduce now a filter $F^{\infty}$, called the fine neighborhood filter of $\infty$, which plays the same role for our Martin boundary point $\infty$, as the corresponding filters in classical potential theory. We start with the following theorem, the first part of which is an analogue of a classical theorem.

(5.2) Theorem. For any Borel set $A \subseteq R^{+}$, either $H_{A} \psi$ is a P-potential or $H_{A} \psi \equiv \psi$. The first case occurs iff $\mu(A)<\infty$.

Since $1-\psi$ is a $P$-potential, $H_{A}(1-\psi)$ is a $P$-potential; therefore $\left(H_{A} 1-H_{A} \psi\right)^{\infty}$ $=0$, i.e., $\left(H_{A} 1\right)^{\infty}=\left(H_{A} \psi\right)^{\infty}$. Hence we can write Theorem (5.2) in the equivalent form:

(5.2') THEOREM. For any Borel set $A \subseteq R^{+}$, either $\left(H_{A} 1\right)^{\infty} \equiv 0$ or $\equiv \psi$. The first case occurs iff $\mu(A)<\infty$. 
Proof of Theorem (5.2'). We shall prove first that $\mu(A)<\infty$ implies $\left(H_{A} 1\right)^{\infty} \equiv 0$. We have $H_{A} 1(\xi) \leqq G(\xi, A)$, which implies that $P_{n} H_{A} 1(\xi) \leqq P_{n} G(\xi, A)=\sum_{k=n}^{\infty} P_{k}(\xi, A)$. Since $\mu(A)<\infty$, we conclude by (3.9) that

$$
G(\xi, A)=\sum_{k=0}^{\infty} P_{k}(\xi, A)<\infty \quad \text { for } \xi \in R^{+} .
$$

We therefore get $\left(H_{A} 1\right)^{\infty}=\lim _{n \rightarrow \infty} P_{n} H_{A} 1 \equiv 0$.

If on the other hand $\mu(A)=\infty$, we shall prove that $\left(H_{A} 1\right)^{\infty} \equiv \psi$. By (2.4) and (4.1) we get $H_{A} 1=\alpha \psi+v$, where $v$ is a $P$-potential. By (4.13) and (4.14) there is a Borel set $B$ such that $\mu(B)<\infty$ and $\lim _{\xi \rightarrow \infty ; \xi \notin B} H_{A} 1(\xi)=\alpha$. Since $H_{A} 1(\xi)=1$ for $\xi \in A$ and $\mu(A)=\infty$, we get $\alpha=1$, therefore $\left(H_{A} 1\right)^{\infty} \equiv \psi$. Q.E.D.

If we now define a set $A \subseteq R^{+}$to be thin at $\infty$ if $\mu^{*}(A)<\infty\left(\mu^{*}=\right.$ outer extension of $\mu$ ), then by Theorem (5.2) a Borel set $A$ is thin at $\infty$ iff $H_{A} \psi$ is a $P$-potential in analogy to a classical theorem. If we define as in the classical case the filter $F^{\infty}$ on $R^{+}$as the class of complements of the sets which are thin at $\infty$, this filter converges to $\infty$ and Corollary (4.16) can be restated in the following equivalent form:

(5.3) ThEOREM. If $u \geqq 0$ is $P$-superregular, then $\lim _{F^{\infty}} u$ exists and is finite.

In view of Theorem (5.1) Theorem (5.3) is the analogue of the Fatou fine limit theorem in classical potential theory. The equivalent form (4.16) has also its counterpart there.

The equivalence of Theorem (5.3) and Corollary (4.16) follows as in the classical case from the following theorem, which has its counterpart in classical potential theory, too, and discusses the relations between "fine limit" concepts at $\infty$ and Martin limit concepts (=ordinary limit concepts as $\xi \rightarrow \infty$ ).

(5.4) TheOREM. Let $A \subseteq R^{+}$be such that $\mu^{*}(A)=\infty$ and let $g$ be an extended real-valued function defined on $A$. Then

(a) There exists a set $B \subseteq A$ such that $\mu^{*}(B)<\infty$ and

$$
\lim _{F^{\infty} \cap A} \sup _{\cap} g=\lim _{\xi \rightarrow \infty ; \xi \in A-B} g(\xi) \text {. }
$$

(b) If $c$ is any fine cluster value of $g$ at $\infty$ (i.e., a cluster value of $g$ with respect to $\left.F^{\infty} \cap A\right)$, there exists a set $A_{0} \subseteq A$ with $\mu^{*}\left(A_{0}\right)=\infty$ such that $\lim _{\xi \rightarrow \infty ; \xi \in A_{0}} g(\xi)$ exists and is equal to $c$.

(It will be clear from the proof that the statements under (a) and (b) will remain true if we replace the sets $A, B, A_{0}$ by Borel sets $A, B, A_{0}$ for which $\mu(A)=\infty$, $\mu(B)<\infty, \mu\left(A_{0}\right)=\infty$ and if we make for (b) the additional assumption that $g$ be Borel measurable.)

Proof of Theorem (5.4). The proof is along the lines of the one in [2] for the classical case. It is of course simpler because of the simple structure of thin sets.

(a) Let $\alpha=\lim \sup _{F^{\infty} \cap A} g$. Since $F^{\infty} \cap A \rightarrow \infty$, we clearly have that $\alpha \leqq \lim \sup _{\xi \rightarrow \infty ; \xi \in A} g(\xi)$. It is therefore sufficient to assume $\alpha<\infty$. If for $\varepsilon>0$, 
we let $B_{\varepsilon}=\{\xi ; \xi \in A, g(\xi)>\alpha+\varepsilon\}$, then $\mu^{*}\left(B_{\varepsilon}\right)<\infty$. Choose $\xi_{n} \uparrow \infty$ such that $\mu^{*}\left\{B_{1 / n} \cap\left(\xi_{n}, \infty\right)\right\}<1 / n^{2}$ and let $B=\bigcup_{n=1}^{\infty}\left\{B_{1 / n} \cap\left(\xi_{n}, \infty\right)\right\}$. Then we have $\mu^{*}(B)<\infty$, and also $g(\xi) \leqq \alpha+1 / n$ for $\xi \in(A-B) \cap\left(\xi_{n}, \infty\right)$, hence $\lim \sup _{\xi \rightarrow \infty ; \xi \in A-B} g(\xi) \leqq \alpha$.

The reversed inequality is obvious. (We recall that in the proof of Theorem (4.13) we actually showed that for a $P$-potential $v, \lim _{F} \infty v=0$, and concluded as above the existence of $B$ such that $\mu(B)<\infty$ and $\lim _{\xi \rightarrow \infty ; \xi \in B^{c}} v(\xi)=0$.)

(b) We assume $-\infty<c<+\infty$. (The changes in the proof required for $c= \pm \infty$ will be obvious.) By definition, $c$ is a cluster value of $g$ with respect to $F^{\infty} \cap A$ if

$$
c \in \bigcap_{A^{\prime} \in F^{\infty} \cap A} \overline{g\left(A^{\prime}\right)}
$$

or equivalently if for any $\varepsilon>0$ and for any $A^{\prime} \in F^{\infty} \cap A$ (i.e., for any set $A^{\prime}$ such that $A^{\prime} \subseteq A$ and $\left.\mu^{*}\left(A-A^{\prime}\right)<\infty\right)$

$$
\left\{\xi ; \xi \in A^{\prime}, c-\varepsilon<g(\xi)<c+\varepsilon\right\} \neq \varnothing .
$$

If for $\varepsilon>0$, we let $A_{\varepsilon}=\{\xi ; \xi \in A, c-\varepsilon<g(\xi)<c+\varepsilon\}$ then $\mu^{*}\left(A_{\varepsilon}\right)=\infty$ because if we had $\mu^{*}\left(A_{\varepsilon}\right)<\infty$, (X) would imply a contradiction for $A^{\prime}=A-A_{\varepsilon}$. Now let $\xi_{1}=0$ and if $\xi_{n}$ is already chosen, choose $\xi_{n+1}>\xi_{n}$ such that $\mu^{*}\left\{A_{1 / n} \cap\left(\xi_{n}, \xi_{n+1}\right]\right\} \geqq 1$ (this implies $\left.\xi_{n} \rightarrow \infty\right)$ and let $A_{0}=\bigcup_{n=1}^{\infty}\left\{A_{1 / n} \cap\left(\xi_{n}, \xi_{n+1}\right]\right\}$. Then $\mu^{*}\left(A_{0}\right)=\infty$, and also $|g(\xi)-c|<1 / n$ for $\xi \in A_{0} \cap\left(\xi_{n}, \infty\right)$, hence $\lim _{\xi \rightarrow \infty ; \xi \in A_{0}} g(\xi)$ exists and is equal to $c$.

In the formulation of (5.3) we have recognized Corollary (4.16) as a Fatou fine limit theorem. We point out that Theorem (4.14) is the analogue of the classical nontangential limit theorem for positive harmonic functions on a ball in $R^{n}$. "Nontangential" approach to $\infty$ is in our case the same as approach to $\infty$ in the Martin topology, i.e., unrestricted approach to $\infty$, since $\infty$ is the only Martin boundary point. Theorem (4.14) was derived in $\S 4$ from the corresponding fine limit theorem, i.e., $\lim _{F}{ }^{\infty} \psi=1$ (or equivalently: there exists $B$ such that $\mu(B)<\infty$ and $\left.\lim _{\xi \rightarrow \infty: \xi \notin B}(1-\psi)(\xi)=0\right)$, and the analogue of the classical Harnack inequality. For the classical theorem the corresponding derivation was given in [3]. We have no counterpart to the classical radial limit theorem for positive superharmonic functions on a ball in $R^{n}$. Corollary (4.15) is the counterpart of a theorem by Tolsted [18], which states the existence of nontangential limits at almost all boundary points for certain averages of a positive superharmonic function on a ball in $R^{n}$.

Just as in the classical case the Fatou fine limit Theorem (5.3) has also a probabilistic interpretation. As we mentioned in $\$ 2$ the process $\left\{X_{\xi n}, n \geqq 0\right\}$ with state space $R^{+} \cup\{\rho\}$, stochastic transition function $P^{\prime}$ (=stochastic extension of $P$ ) and starting point $\xi$, plays in our potential theory the same role as Brownian motion in classical potential theory.

We mentioned in $\$ 2$ that the supermartingale convergence theorem implies the following theorem:

(5.5) THEOREM. If $u \geqq 0$ is $P$-superregular, then for all $\xi \in R^{+}, \lim _{n \rightarrow \infty} u^{\prime}\left(X_{\xi n}\right)$ exists and is finite $\mathrm{Pr}_{\xi}$-a.e. 
We shall prove below the much stronger Theorem (5.8), in which we actually identify the limit. It is clear that for this purpose we may restrict our attention to paths converging to $\infty$, because if $\zeta_{\xi}<\infty$, then $u^{\prime}\left(X_{\xi n}\right)=0$ except for finitely many $n$.

We turn now to the probabilistic characterization of Borel sets which are thin at $\infty$. The characterization is actually given by $\left(5.2^{\prime}\right)$, which reads in probabilistic notation:

(5.0) 0-1-law. For any Borel set $A \subseteq R^{+}, \operatorname{Pr}_{\xi}\left\{X_{\xi n} \in A\right.$ i.o. $\} \equiv 0$ or $\equiv \psi$. The first case occurs iff $\mu(A)<\infty$ (i.e., $A$ is thin at $\infty$ ).

This 0-1-law leads to the following

(5.7) THEOREM. Let $g$ be an extended real-valued Borel function on $R^{+}$. Then for all $\xi \in R^{+}$, $\lim \sup _{n \rightarrow \infty} g\left(X_{\xi n}\right)=\lim \sup _{F^{\infty}} g \operatorname{Pr}_{\xi}$-a.e. on $\Lambda_{\xi}$, where $\Lambda_{\xi}=\left\{\omega_{\xi}\right.$; $\left.X_{\xi n}\left(\omega_{\xi}\right) \rightarrow \infty\right\}$.

Proof. We shall use Theorem (5.4) as modified for measurable $A$ and $g$.

Let $\alpha=\lim \sup _{F^{\infty}} g$. By Theorem (5.4) (a) there exists a Borel set $B$ such that $\mu(B)<\infty$ and lim $\sup _{\xi \rightarrow \infty ; \xi \notin B} g(\xi)=\alpha$. By (5.6) we conclude that $\operatorname{Pr}_{\xi}\left\{X_{\xi n} \in B\right.$ i.o. $\}$ $\equiv 0$; this implies that $\lim \sup _{n \rightarrow \infty} g\left(X_{\xi n}\right) \leqq \alpha \operatorname{Pr}_{\xi}$-a.e. on $\Lambda_{\xi}$. On the other hand, since $\alpha$ is a fine cluster value of $g$ at $\infty$, we know by Theorem (5.4) (b) that there exists a Borel set $A_{0}$ such that $\mu\left(A_{0}\right)=\infty$ and $\lim _{\xi \rightarrow \infty: \xi \in A_{0}} g(\xi)=\alpha$. By (5.6) we conclude that $\operatorname{Pr}_{\xi}\left\{X_{\xi n} \in A_{0}\right.$ i.o. $\} \equiv \psi(\xi)$, i.e., $\left\{X_{\xi n} \in A_{0}\right.$ i.o. $\}=\Lambda_{\xi} \operatorname{Pr}_{\xi}$-a.e.; this implies that $\lim \sup _{n \rightarrow \infty} g\left(X_{\xi n}\right) \geqq \alpha \operatorname{Pr}_{\xi}$-a.e. on $\Lambda_{\xi}$.

REMARK. Theorem (5.7) says-apart from the identification of the limit-that for all $\xi \in R^{+}$, lim sup $\operatorname{su}_{n \rightarrow \infty} g\left(X_{\xi n}\right)$ is a constant $\operatorname{Pr}_{\xi}$-a.e. on $\Lambda_{\xi}$ and that this constant, defined for all $\xi \in R^{+}$such that $\psi(\xi)>0$, is independent of $\xi$. This is a special situation of a 0-1-law which appears formally stronger than (5.6) and which we shall give in $\$ 6$.

Theorem (5.7) allows us to give a probabilistic version of Theorem (5.3):

(5.8) TheOREM. If $u \geqq 0$ is $P$-superregular and $\lim _{F^{\infty}} u=\alpha$, then for all $\xi \in R^{+}$, $\lim _{n \rightarrow \infty} u\left(X_{\xi n}\right)=\alpha, \operatorname{Pr}_{\xi}$-a.e. on $\Lambda_{\xi}$.

We see that-and in this aspect the classical case is different-we need not consider the process, "conditioned to go to $\infty$," for the probabilistic interpretation of thin sets and fine limit concepts, since the $\operatorname{Pr}_{\xi}$-measure of the set of $X_{\xi n}$-paths, which converge to our only Martin boundary point $\infty$, equals $\psi(\xi)$ and we assumed $\psi \not \equiv 0$. If $\psi \not \equiv 0$ we actually know from $\S 3$, that the set of positivity of $\psi$, which we shall denote by $I$, is an interval $(a, \infty)$ or $[a, \infty)$ where $a \in R^{+}$. For $\xi \in I$ we could of course define a process $X_{\xi n}^{\infty}$-in analogy to the classical case-on some probability space $\left(\Omega_{\xi}^{\infty}, \mathfrak{A}_{\xi}^{\infty}, \operatorname{Pr}_{\xi}^{\infty}\right)$ with state space $I$, starting point $\xi$ and transition function

$$
P^{\infty}\left(\xi, d \xi^{\prime}\right)=\frac{\psi\left(\xi^{\prime}\right)}{\psi(\xi)} P\left(\xi, d \xi^{\prime}\right)
$$


(We distinguish all symbols referring to this process by a superscript " $\infty$.") Since $P^{\infty}$ is stochastic, we have for the life-time $\zeta_{\xi}^{\infty}=\infty \operatorname{Pr}_{\xi}^{\infty}$-a.e. Since

$$
G^{\infty}(\xi, A)=\sum_{n=0}^{\infty} \operatorname{Pr}_{\xi}^{\infty}\left\{X_{\xi n}^{\infty} \in A\right\}=\int_{A} \frac{\psi\left(\xi^{\prime}\right)}{\psi(\xi)} G\left(\xi, d \xi^{\prime}\right)
$$

is finite for bounded $A$, we have

$$
\lim _{n \rightarrow \infty} X_{\xi n}^{\infty}=\infty \operatorname{Pr}_{\xi}^{\infty} \text {-a.e. }
$$

Now we can modify (5.6) to the following complete analogue of the classical case:

(5.6') 0-1-law. For any Borel set $A \subseteq R^{+}$, we have on I

$$
\operatorname{Pr}_{\xi}\left\{X_{\xi n}^{\infty} \in A \text { i.o. }\right\} \equiv 0 \text { or } \equiv 1 \text {. }
$$

The first case occurs iff $\mu(A)<\infty$ (i.e., $A$ is thin at $\infty$ ).

Proof. We have $P_{n}^{\infty} H_{A}^{\infty} 1(\xi)=P_{n} H_{A} \psi(\xi) / \psi(\xi)$ for $\xi \in I$, which implies together with Theorem (5.2) that $\lim _{n \rightarrow \infty} P_{n}^{\infty} H_{A}^{\infty} 1(\xi) \equiv 0$ or $\equiv 1$ on $I$ according to whether $\mu(A)<\infty$ or not. Q.E.D.

We can modify (5.8) in complete analogy of the classical case to:

(5.8') TheOREM. If $u \geqq 0$ is P-superregular and $\lim _{F^{\infty}} u=\alpha$, then for $\xi \in I$, $\lim _{n \rightarrow \infty} u\left(X_{\xi n}^{\infty}\right)=\alpha, \operatorname{Pr}_{\xi}^{\infty}-$ a.e.

6. Supplements. (1) Existence of $\lim _{\eta \rightarrow \infty} g(\xi, \eta)$. Under certain conditions we can replace the assertion in Theorem (5.1) by a stronger one.

(6.1) THEOREM. Let $\mu_{1}$ be the measure obtained from $\mu$ through translation by 1 , i.e., for any Borel set $A \subseteq R^{+}, \mu_{1}(A)=\mu(A+1)$. If $\lim _{\eta \rightarrow \infty}\left|\mu-\mu_{1}\right|(\eta, \eta+1]=0$, then $\lim _{n \rightarrow \infty} g(\xi, \eta)$ exists for $\xi \in R^{+}$.

Proof. We have $G(\xi, A)=\int_{A} g\left(\xi, \eta^{\prime}\right) \mu\left(d \eta^{\prime}\right)+\chi_{A}(\xi)$, for any Borel set $A$. If we let $A=[\eta-1, \eta)$ in this formula, we get

$$
g(\xi, \eta)=\int_{[\eta-1, \eta)} g\left(\xi, \eta^{\prime}\right) \mu\left(d \eta^{\prime}\right)+\chi_{(\xi, \xi+1]}(\eta) .
$$

The existence of $\lim _{\eta \rightarrow \infty} g(\xi, \eta)$ for $\xi \in R^{+}$follows now from a theorem in [16], the version of which in our context asserts the existence of $\lim _{n \rightarrow \infty} h(\eta)$ for any bounded function $h$ which satisfies $h(\eta)=\int_{[\eta-1, \eta)} h\left(\eta^{\prime}\right) \mu\left(d \eta^{\prime}\right)$ for $\eta>A$, under the condition $\lim _{\eta \rightarrow \infty}\left|\mu-\mu_{1}\right|(\eta, \eta+1]=0$.

(6.3) RemarK. If $\mu(d \xi)=K(\xi) d \xi$ and $K \leqq 1$, the condition that

$$
\lim _{\xi \rightarrow \infty}\left|\mu-\mu_{1}\right|(\xi, \xi+1]=0
$$

is satisfied if $\int_{0}^{\infty}\{1-K(\xi)\} d \xi<\infty$ because

$$
\left|\mu-\mu_{1}\right|(\xi, \xi+1]=\int_{\xi}^{\xi+1}\left|K\left(\xi^{\prime}+1\right)-K\left(\xi^{\prime}\right)\right| d \xi^{\prime} \leqq \int_{\xi}^{\xi+2}\left\{1-K\left(\xi^{\prime}\right)\right\} d \xi^{\prime} .
$$

By (4.7) the condition $\int_{0}^{\infty}\{1-K(\xi)\} d \xi<\infty$ is equivalent to $\psi \not \equiv 0$. 
(6.4) Remark. In the nondefective case $\mu(\xi, \xi+1] \equiv 1, \mu$ is determined by its values in one interval $(\xi, \xi+1]$, and we have $\mu \equiv \mu_{1}$. In this case the condition of Theorem (6.1) is trivially satisfied.

As for the identification of the limit in Theorem (6.1) we shall prove

(6.5) THEOREM. If for some $\xi_{0} \in R^{+}, \psi\left(\xi_{0}\right)>0$ and $\lim _{\eta \rightarrow \infty} g\left(\xi_{0}, \eta\right)$ exists, then $\alpha=\lim _{\xi \rightarrow \infty} \int_{(\xi, \xi+1]} \mu\{\eta\} \mu(d \eta)$ exists and $\lim _{\eta \rightarrow \infty} g(\xi, \eta)=2 \psi(\xi) /(1+\alpha)$ for all $\xi \in R^{+}$.

Proof. Under the assumptions it is clear from Theorem (5.1) that $\lim _{n \rightarrow \infty} g(\xi, \eta)$ exists for all $\xi \in R^{+}$and is equal to $a \cdot \psi(\xi)$. In order to identify $a$ we apply formula (2.6) to $A=(\eta, \eta+1]$ and get:

$$
H_{(\eta, \eta+1]} 1(\eta)=\int\left\{H_{(\eta, n+1]} 1-P H_{(\eta, \eta+1]} 1\right\}\left(\eta^{\prime}\right) G\left(\xi, d \eta^{\prime}\right) .
$$

Since

we get for $\xi \leqq \eta$

$$
\begin{aligned}
\left\{H_{(\eta, \eta+1]} 1-P H_{(\eta, \eta+1]} 1\right\}\left(\eta^{\prime}\right) & =0 \text { for } n^{\prime} \notin(\eta, \eta+1], \\
& =1-\mu\left(\eta^{\prime}, \eta+1\right] \text { for } \eta^{\prime} \in(\eta, \eta+1],
\end{aligned}
$$

$$
H_{(\eta, \eta+1]} 1(\xi)=\int_{(\eta, \eta+1]}\left\{1-\mu\left(\eta^{\prime}, \eta+1\right]\right\} g\left(\xi, \eta^{\prime}\right) \mu\left(d \eta^{\prime}\right) .
$$

Together with $\lim _{\eta \rightarrow \infty} g(\xi, \eta)=a \psi(\xi)$, this implies that for any $\varepsilon>0$, there is an $\eta_{\varepsilon}$ such that for $\eta>\eta_{\varepsilon}$

where

$$
a \psi(\xi) \cdot I(\eta)-\varepsilon \leqq H_{(\eta, \eta+1]} 1(\xi) \leqq a \psi(\xi) \cdot I(\eta)+\varepsilon,
$$

$$
I(\eta)=\int_{(\eta, \eta+1]}\left\{1-\mu\left(\eta^{\prime}, \eta+1\right]\right\} \mu\left(d \eta^{\prime}\right)
$$

Since by (4.10) $\lim _{n \rightarrow \infty} H_{(\eta, n+1]} 1(\xi)=\psi(\xi)$, we conclude that

$$
\lim _{\eta \rightarrow \infty} I(\eta)=\frac{1}{a}
$$

We have:

(1) $\lim _{n \rightarrow \infty} \int_{(n, \eta+1]} \mu\left(d \eta^{\prime}\right)=1$

and we shall prove below that

(2) $\int_{(\eta, \eta+1]} \mu\left(\eta^{\prime}, \eta+1\right] \mu\left(d \eta^{\prime}\right)=\frac{1}{2}\left\{(\mu(\eta, \eta+1])^{2}-\int_{(\eta, \eta+1]} \mu\left\{\eta^{\prime}\right\} \mu\left(d \eta^{\prime}\right)\right\}$.

Then the existence of $\lim _{\eta \rightarrow \infty} I(\eta)$ and (1) imply the existence of

$$
\lim _{\eta \rightarrow \infty} \int_{(\eta, n+1]} \mu\left(\eta^{\prime}, \eta+1\right] \mu\left(d \eta^{\prime}\right),
$$

which together with (1) and (2) implies the existence of $\lim _{\eta \rightarrow \infty} \int_{(\eta, \eta+1]} \mu\left\{\eta^{\prime}\right\} \mu\left(d \eta^{\prime}\right)$. Moreover we get:

$$
\lim _{\eta \rightarrow \infty} I(\eta)=\frac{1}{2}\left\{1+\lim _{\eta \rightarrow \infty} \int_{(\eta, n+1]} \mu\left\{\eta^{\prime}\right\} \mu\left(d \eta^{\prime}\right)\right\} .
$$


We still have to show (2), which is done by applying Fubini's theorem:

$$
\begin{aligned}
\int_{(\eta, \eta+1]} \mu\left(\eta^{\prime}, \eta+1\right] \mu\left(d \eta^{\prime}\right) & =\int_{(\eta, \eta+1]} \int_{\left(\eta^{\prime}, \eta+1\right]} \mu\left(d \eta^{\prime \prime}\right) \mu\left(d \eta^{\prime}\right) \\
& =\int_{(\eta, \eta+1]} \int_{\left(\eta, \eta^{\prime \prime}\right)} \mu\left(d \eta^{\prime}\right) \mu\left(d \eta^{\prime \prime}\right) \\
& =\int_{(\eta, \eta+1]} \mu\left(\eta, \eta^{\prime}\right) \mu\left(d \eta^{\prime}\right),
\end{aligned}
$$

which implies (2).

(2) 0-1-law. Let $\left\{Y_{n}, n \geqq 0\right\}$ be a sequence of measurable functions from a measurable space $\left(\Omega_{1}, \mathfrak{A}_{1}\right)$ into a measurable space $\left(\Omega_{2}, \mathfrak{A}_{2}\right)$. Let $\mathfrak{B}\left(Y_{n}, Y_{n+1}, \ldots\right)$ denote the smallest sub- $\sigma$-field of $\mathfrak{A}_{1}$, with respect to which the functions $Y_{n}$, $Y_{n+1} \ldots$ are measurable. The $\mathfrak{B}\left(Y_{n}, Y_{n+1}, \ldots\right)$ form a nonincreasing sequence and its limit $\mathfrak{B}_{\infty}\left(Y_{0}, Y_{1}, \ldots\right)=\bigcap_{n=0}^{\infty} \mathfrak{B}\left(Y_{n}, Y_{n+1}, \ldots\right)$ is called the tail-field of the sequence $\left\{Y_{n}, n \geqq 0\right\}$. A set in $\mathfrak{B}_{\infty}\left(Y_{0}, Y_{1}, \ldots\right)$ is called a tail set of $\left\{Y_{n}, n \geqq 0\right\}$, an extended real-valued function on $\Omega_{1}$ which is $\mathfrak{B}_{\infty}\left(Y_{0}, Y_{1}, \ldots\right)$-measurable, a tail function of $\left\{Y_{n}, n \geqq 0\right\}$.

Now let $\Omega_{2}=R^{+} \cup\{\rho\}$ and $\mathfrak{A}_{2}=$ Borel field of $R^{+} \cup\{\rho\}$; let $\left(\Omega_{1}, \mathfrak{A}_{1}\right)$ be the corresponding sequence space, i.e., $\Omega_{1}=\Omega_{2}^{T}, \mathfrak{A}_{1}=\mathfrak{A}_{2}^{T}$ where $T=\{0,1,2, \ldots\}$; then for $n \geqq 0$ the functions $X_{n}: \Omega_{1} \rightarrow \Omega_{2}$, defined by: $X_{n}(\omega)=n$th coordinate of $\omega$, for $\omega \in \Omega_{1}$, are measurable. Now any tail function $f$ of $\left\{X_{n}, n \geqq 0\right\}$ determines for each $\xi \in R^{+} \cup\{\rho\}$ a tail function $f_{\xi}$ of $\left\{X_{\xi n}, n \geqq 0\right\}$ by

$$
f_{\xi}\left(\omega_{\xi}\right)=f\left(X_{\xi 0}\left(\omega_{\xi}\right), X_{\xi 1}\left(\omega_{\xi}\right), \ldots\right) \text { for } \omega_{\xi} \in \Omega_{\xi},
$$

and any tail set $B$ of $\left\{X_{n}, n \geqq 0\right\}$ determines for each $\xi \in R^{+} \cup\{\rho\}$ a tail set $B_{\xi}$ of $\left\{X_{\xi n}, n \geqq 0\right\}$ by

$$
\chi_{B_{\xi}}\left(\omega_{\xi}\right)=\left(\chi_{B}\right)_{\xi}\left(\omega_{\xi}\right) \text { for } \omega_{\xi} \in \Omega_{\xi} .
$$

E.g., for the tail set $\Lambda=\left\{\omega ; X_{n}(\omega) \rightarrow \infty\right\}$ of $\left\{X_{n}, n \geqq 0\right\}$, we get the tail set $\Lambda_{\xi}$ $=\left\{\omega_{\xi} ; X_{\xi n}\left(\omega_{\xi}\right) \rightarrow \infty\right\}$ of $\left\{X_{\xi n}, n \geqq 0\right\}$.

(6.7) 0-1-law. Let $B$ be a tail set of $\left\{X_{n}, n \geqq 0\right\}$. Then either (1) or (2) is true:

(1) $\Lambda_{\xi} \subseteq B_{\xi}, \operatorname{Pr}_{\xi}$-a.e., i.e., $\operatorname{Pr}_{\xi}\left\{B_{\xi} \cap \Lambda_{\xi}\right\}=\psi(\xi)$ for $\xi \in R^{+}$,

(2) $\Lambda_{\xi} \cap B_{\xi}=\varnothing, \operatorname{Pr}_{\xi}$-a.e., i.e., $\operatorname{Pr}_{\xi}\left\{B_{\xi} \cap \Lambda_{\xi}\right\}=0$ for $\xi \in R^{+}$.

Proof. For $\xi \in R^{+} \cup\{\rho\}$, let $h(\xi)=\operatorname{Pr}_{\xi}\left\{B_{\xi} \cap \Lambda_{\xi}\right\}$. Since $P^{\prime}(\cdot, A)$ is measurable for each $A$ in the Borel field of $R^{+} \cup\{\rho\}, h$ is measurable. From

$$
\begin{aligned}
h\left(X_{\xi n}\right) & =\operatorname{Pr}_{X_{\xi}}\left\{B_{X_{\xi n}} \cap \Lambda_{X_{\xi n}}\right\}=\operatorname{Pr}_{\xi}\left\{B_{\xi} \cap \Lambda_{\xi} \mid X_{\xi n}\right\} \\
& =\operatorname{Pr}_{\xi}\left\{B_{\xi} \cap \Lambda_{\xi} \mid X_{\xi 0}, X_{\xi 1}, \ldots, X_{\xi n}\right\},
\end{aligned}
$$

we get by Lévy's Theorem on successive conditional probabilities

$$
\lim _{n \rightarrow \infty} h\left(X_{\xi n}\left(\omega_{\xi}\right)\right)=\chi_{B_{\xi} \cap \Lambda_{\xi}}\left(\omega_{\xi}\right), \quad \operatorname{Pr}_{\xi} \text {-a.e. }
$$


By the 0-1-law (5.6) we know:

$$
\operatorname{Pr}_{\xi}\left\{\omega_{\xi} ; \lim _{n \rightarrow \infty} h\left(X_{\xi n}\left(\omega_{\xi}\right)\right)=1\right\} \equiv 0 \quad \text { or } \equiv \psi(\xi) .
$$

(6.8) and (6.9) prove the 0-1-law (6.7).

(6.10) Corollary. Let $f$ be a tail function of $\left\{X_{n}, n \geqq 0\right\}$. Then for $\xi \in R^{+}, f_{\xi}$ is a constant on $\Lambda_{\xi}, \operatorname{Pr}_{\xi}$-a.e. Moreover, this constant, defined for all $\xi$ for which $\psi(\xi)>0$, is independent of $\xi$.

Proof. Let $A$ be any Borel set of the extended real line. Applying (6.7) to the tail set $B=\{\omega ; f(\omega) \in A\}$ of $\left\{X_{n}, n \geqq 0\right\}$ we get that either $\left(1^{\prime}\right)$ or $\left(2^{\prime}\right)$ is true:

(1') $\Lambda_{\xi} \subseteq\left\{\omega_{\xi} ; f_{\xi}\left(\omega_{\xi}\right) \in A\right\}, \operatorname{Pr}_{\xi}$-a.e. for $\xi \in R^{+}$,

(2') $\Lambda_{\xi} \subseteq\left\{\omega_{\xi} ; f_{\xi}\left(\omega_{\xi}\right) \in A^{c}\right\}, \operatorname{Pr}_{\xi}$-a.e. for $\xi \in R^{+}$.

This proves the corollary.

(3) The nondefective case $\mu(\xi, \xi+1] \equiv 1$ and random walk. In the nondefective case $\mu(\xi, \xi+1] \equiv 1$ (i.e., if $P$ is stochastic or equivalently if $\psi \equiv 1$ ) we have a Markov process on $R^{+}$with transition function $P$ and starting point $\xi \in R^{+}$. For this process $X_{\xi n}<X_{\xi, n+1} \operatorname{Pr}_{\xi}$-a.e. Such processes are sometimes called "positive processes." A "positive process" with state space $\mathfrak{X}=\{0,1,2, \ldots\}$ is the following "random walk" $Z_{\text {in }}$ :

Let $Y_{1}$ be a random variable, taking values $1,2,3, \ldots$ with probability $p(j)>0$, such that $E Y_{1}<\infty$. Let $P^{*}(i, j)=p(j-i)$; then $P^{*}$ is a stochastic transition function on $\mathfrak{X}$. We shall distinguish all symbols referring to this transition function by “*”. Let $\left\{Z_{i n}, n \geqq 0\right\}$ be the Markov process with starting point $i$ and transition function $P^{*}$. Clearly for $n \geqq 1$ :

$$
Z_{\text {in }}=i+Y_{1}+Y_{2}+\cdots+Y_{n},
$$

where the $Y_{i}$ are independent random variables with the same distribution as $Y_{1}$. The process $\left\{Z_{\text {in }}\right\}$ is a special case of a class of Markov processes, the so-called random walks. Their potential theory is discussed in [8], [17].

We shall point out now that the probabilistic structures of the processes $\left\{X_{\xi n}, n>0\right\}$ and $\left\{Z_{i n}, n \geqq 0\right\}$ and equivalently the structures of the corresponding potential theories are very similar.

(a) Potential theory. By Remark (6.4) and Theorem (6.5) we have

$$
\lim _{\eta \rightarrow \infty} g(\xi, \eta)=2 /\left(1+\int_{(0,1]} \mu\{\eta\} \mu(d \eta)\right) .
$$

This is the analogue of the renewal theorem (see e.g., [17]) for the $Z_{\text {in }}$ process:

$$
\lim _{j \rightarrow \infty} g^{*}(i, j)=\frac{1}{E Y_{1}}
$$

(6.12) and (6.13) imply that $\lim _{\eta \rightarrow \infty} k(\eta, \xi)=1$ and $\lim _{j \rightarrow \infty} k^{*}(j, i)=1$. Therefore in both cases the one-point-compactification of the state space can be considered 
as Martin space and the Alexandroff point $\infty$ as Martin boundary point. In both cases the nonnegative regular functions are the nonnegative constants, i.e., 1 can be considered as minimal function, corresponding to the boundary point $\infty$.

From the renewal theorem (6.13) one can easily deduce that for a $P^{*}$-potential $v$

$$
\lim _{i \rightarrow \infty} v(i)=0 \text {. }
$$

This is the analogue of Theorem (4.13), because by a result from [17] for the $P^{*}$-potential theory the finite sets play the role of the sets of finite $\mu$-measure in the $P$-potential theory, i.e., they are the transient sets as well as the sets which are thin at $\infty$.

Remark. In [17], a set $A$ is defined to be transient if

$$
H_{A} 1(\xi)<1 \text { for some } \xi \in \mathfrak{X} .
$$

Since $(\mathrm{X})$ is true iff $\left(H_{A} 1\right)^{\infty}(\xi) \equiv 0$, this definition is superficially closer to our definition of sets which are thin at $\infty$.

(b) Probability theory. (1) In our present case we can reformulate the 0-1-law (6.7), which we derived for the $X_{\xi n}$-process, to

(6.7') 0-1-law. For any tail set $B$, either $\operatorname{Pr}_{\xi}\left(B_{\xi}\right) \equiv 1$ or $\equiv 0$.

The corresponding 0 -1-law is true for the process $\left\{Z_{i n}, n \geqq 0\right\}$.

The fact that for fixed $i, \operatorname{Pr}_{i}^{*}\left(B_{i}\right)=1$ or $=0$ follows from a theorem by Hewitt and Savage [10]. The fact that the value of $\operatorname{Pr}_{i}^{*}\left(B_{i}\right)$ is independent of $i$ follows easily as in the case we discussed.

(2) The statement that thin sets and transient sets coincide for the processes $X_{\xi n}$ and $Z_{i n}$, is another way of saying that the Borel-Cantelli Lemma holds for the events $\left\{X_{\xi n} \in A\right\}$ and $\left\{Z_{\text {in }} \in A\right\}$.

(3) Whereas (6.11) represents $Z_{\text {in }}$ as the sum of the first $n$ elements of a sequence of independent, identically distributed (disregarding the first term) random variables, we show now that one can represent $X_{\xi n}$ as the sum of the first $n$ elements of a sequence of random variables, which have the same distribution (except for the first one) and two of which are independent if there is at least one between them. This is a consequence of $\mu=\mu_{1}$ (Remark (6.4)) and can be seen as follows:

We may assume $\xi=0$. Then

$$
X_{0 n}=X_{01}+\left(X_{02}-X_{01}\right)+\cdots+\left(X_{0 n}-X_{0, n-1}\right) .
$$

Now if we let $\theta_{i}=X_{0 i}-\left[X_{0 i}\right]$, where $[a]=n$ if $n<a \leqq n+1$, then the $\theta_{i}$ are independent random variables taking values in $(0,1]$ and having as common distribution the restriction of $\mu$ to $(0,1]$. Moreover,

$$
\begin{aligned}
X_{0, i+1}-X_{0 i} & =\theta_{i+1}-\theta_{i} \quad \text { if } \theta_{i}<\theta_{i+1} \\
& =1+\left(\theta_{i+1}-\theta_{i}\right) \quad \text { if } \theta_{i} \geqq \theta_{i+1} .
\end{aligned}
$$

This implies that the summands in (6.15) (except for the first one) have the same distribution and that any two of them are independent, if there is at least one between them. 
(4) To complete the analogy between (6.12) and (6.13) we shall prove now

$$
E\left(X_{\xi 2}-X_{\xi 1}\right)=\frac{1}{2}\left\{1+\int_{(0,1]} \mu\{\eta\} \mu(d \eta)\right\}
$$

Let us assume again $\xi=0$. Then

hence

$$
E\left\{X_{02}-X_{01} \mid X_{01}\right\}=\int_{\left(X_{01}, x_{01}+1\right]}\left(\xi-X_{01}\right) \mu(d \xi)
$$

$$
\begin{aligned}
E\left(X_{02}-X_{01}\right) & =\int_{(0,1]} \mu(d \eta) \int_{(\eta, \eta+1]}(\xi-\eta) \mu(d \xi) \\
& =\int_{(0,1]}\left(\int_{(\eta, \eta+1]} \xi \mu(d \xi)\right) \mu(d \eta)-\int_{(0,1]} \eta \mu(d \eta) .
\end{aligned}
$$

The first integral is, by Fubini's theorem,

$$
\begin{aligned}
\int_{(0,1]} \xi \mu(0, \xi) \mu(d \xi)+\int_{(1,2]} \xi \mu[\xi-1,1] \mu(d \xi) \\
=\int_{(0,1]} \xi \mu(0, \xi) \mu(d \xi)+\int_{(0,1]}\left(\xi^{\prime}+1\right) \mu\left[\xi^{\prime}, 1\right] \mu\left(d \xi^{\prime}\right) \\
=\int_{(0,1]} \xi \mu(d \xi)+\int_{(0,1]} \mu[\xi, 1] \mu(d \xi) .
\end{aligned}
$$

Therefore $E\left(X_{02}-X_{01}\right)=\int_{(0,1]} \mu[\xi, 1] \mu(d \xi)$. By Fubini's theorem this is equal to $\int_{(0,1]} \mu(0, \xi] \mu(d \xi)$ and therefore equal to $\frac{1}{2}\left\{1+\int_{(0,1]} \mu\{\xi\} \mu(d \xi)\right\}$. (6.15) and (6.16) imply the strong law of large numbers:

$$
X_{\xi n} / n \rightarrow \frac{1}{2}\left\{1+\int_{(0,1]} \mu\{\xi\} \mu(d \xi)\right\}, \quad \operatorname{Pr}_{\xi} \text {-a.e., }
$$

which sharpens our previous result $X_{\xi n} \rightarrow \infty, \operatorname{Pr}_{\xi}$-a.e.

7. The dual problem. The so-called dual problem is concerned with the study of $P$-regular and $P$-superregular measures. Let $\mathfrak{X}$ be a topological space, $\mathfrak{A}$ its Borel field and $P$ a substochastic transition function on $(\mathfrak{X}, \mathfrak{A})$. A measure $\nu$ on $\mathfrak{A}$ is called $P$-regular if

(1) $\nu(A)$ is finite for compact $A$,

(2) $\nu P(A)=\nu(A)$ for $A \in \mathfrak{A}$,

where $\nu P(A)=\int P(\xi, A) \nu(d \xi)$.

$\nu$ is called $P$-superregular if (1) holds and also

(2') $\nu P(A) \leqq \nu(A)$ for $A \in \mathfrak{A}$.

For the Green function $G$ we have: For fixed $\xi \in \mathfrak{X}, G(\xi, \cdot)$ always satisfies (2') and is hence a $P$-superregular measure if $G(\xi, A)<\infty$ for compact $A$.

In this section we shall assume that $\mu$ is a measure on the Borel sets of $R$, the real line, rather than of $R^{+}$; let $P$ denote the transition function on $\left(R, \mathfrak{A}_{R}\right)$ defined by

$$
P(\xi, A)=\mu\{(\xi, \xi+1] \cap A\}, \quad \xi \in R, A \in \mathfrak{A}_{R} .
$$


It is clear immediately that any $P$-regular measure $v$ is absolutely continuous with respect to $\mu$, i.e., that $\mu(A)=0$ implies $\nu(A)=0$. Now let $f$ be the density with respect to $\mu$ of a $P$-regular measure $\nu$. Then we get, by applying Fubini's theorem,

$$
\int_{[\xi-1, \xi)} f(\eta) \mu(d \eta)=f(\xi), \quad \mu \text {-a.e. }
$$

If we denote by $Q$ the transition function on $\left(R, \mathfrak{A}_{R}\right)$ defined by

$$
Q(\xi, A)=\mu\{[\xi-1, \xi) \cap A\}, \quad \xi \in R, A \in \mathfrak{A}_{R},
$$

then $\nu[\xi-1, \xi)=Q f(\xi)$ is a $Q$-regular density of $\nu$ with respect to $\mu$, and in fact is the only such density since two $Q$-regular functions coincide, if they coincide $\mu$-a.e. Conversely if $f \geqq 0$ is $Q$-regular, then the measure $\nu(A)=\int_{A} f(\eta) \mu(d \eta)$ is $P$-regular. We therefore have a $1-1$ correspondence between $P$-regular measures and nonnegative $Q$-regular functions. We get: If $\lim \sup _{\xi \rightarrow-\infty} \mu[\xi-1, \xi)>0$ and $\int_{(-\infty, 0)}\{1-\mu[\xi-1, \xi)\} \mu(d \xi)<\infty$, there is a nontrivial (i.e., $\left.\not \equiv 0\right) P$-regular measure and all $P$-regular measures are proportional.

If $\mu(-\infty, 0)=0$, then all nonnegative $Q$-regular functions are $\equiv 0$; hence the only $P$-regular measure is the trivial measure. This implies that in the potential theory on $R^{+}$, which we considered in the preceding sections, the only $P$-regular measure is the trivial measure.

In the same way as in the regular case we get a 1-1 correspondence between the $P$-superregular measures, which are absolutely continuous with respect to $\mu$ and the nonnegative $Q$-superregular functions; the latter ones are densities of the former ones. E.g., in the case of the $P$-superregular measure $G(\xi, \cdot)$, its density $g(\xi, \cdot)$ is $Q$-superregular, as we see also immediately from (6.2).

We conclude with the remark that $Q$ can be interpreted probabilistically as transition function of the "reversed process."

\section{BIBLIOGRAPHY}

1. M. Brelot, Eléments de la théorie classique du potentiel, Les cours de Sorbonne, Paris, 1959.

2. Introduction axiomatique de l'effilement, Ann. Mat. Pure Appl. 57 (1962), 77-96.

3. M. Brelot and J. L. Doob, Limites angulaires et limites fines, Ann. Inst. Fourier (Grenoble) 13 (1963), 395-415.

4. J. L. Doob, Semimartingales and subharmonic functions, Trans. Amer. Math. Soc. 77 (1954), 86-121.

5. - Conditional Brownian motion and the boundary limits of harmonic functions, Bull. Soc. Math. France 85 (1957), 431-458.

6. - Discrete potential theory and boundaries, J. Math. Mech. 8 (1959), 433-458.

7. - A non-probabilistic proof of the relative Fatou theorem, Ann. Inst. Fourier (Grenoble) 9 (1959), 293-300.

8. J. L. Doob, J. L. Snell and R. E. Williamson, Applications of boundary theory to sums of independent random variables, Contributions to Probability and Statistics, Stanford Univ. Press, Stanford, Calif., 1960, pp. 182-197.

9. E. B. Dynkin, Markov processes, Springer, Berlin, 1965. 
10. E. Hewitt and L. J. Savage, Symmetric measures on Cartesian products, Trans. Amer. Math. Soc. 80 (1955), 470-501.

11. G. A. Hunt, Markov processes and potentials. I, II, III, Illinois J. Math. 1 (1957), 44-93; ibid. 316-369; 2 (1958), 151-213.

12. M. Loève, Probability theory, Van Nostrand, New York, 1955.

13. R. S. Martin, Minimal positive harmonic functions, Trans. Amer. Math. Soc. 49 (1941), 137-172.

14. L. Naim, Sur la rôle de la frontière de R. S. Martin dans la théorie du potentiel, Ann. Inst. Fourier (Grenoble) 7 (1957), 183-281.

15. G. Plackzek, On the theory of slowing down of neutrons in heavy substances, Phys. Rev. 69 (1946), 423-438.

16. M. L. Slater and H. S. Wilf, A class of linear differential-difference equations, Pacific $\mathrm{J}$. Math. 10 (1960), 1419-1427.

17. F. Spitzer, Principles of random walk, Van Nostrand, Princeton, N. J., 1964.

18. E. Tolsted, Non-tangential limits of subharmonic functions, Proc. London Math. Soc. 7 (1957), 321-333.

19. T. Watanabe, A probabilistic method in Hausdorff moment problem and Laplace-Stieltjes transform, J. Math. Soc. Japan 12 (1960), 192-206.

\section{UNIVERSITY OF BRITISH COLUMBIA,}

Vancouver, B. C., Canada 